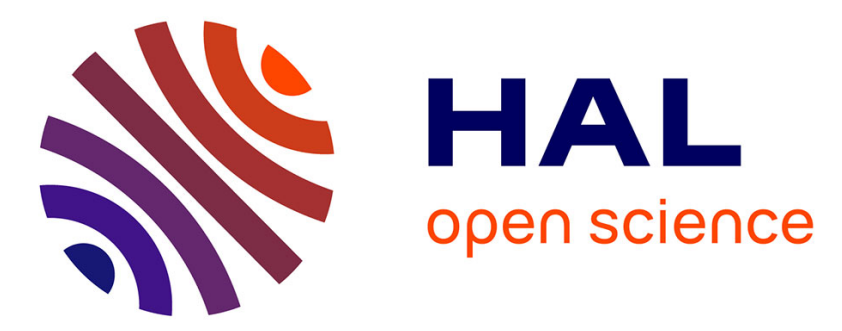

\title{
Fractional-step methods and finite elements with symmetric stabilization for the transient Oseen problem
}

Erik Burman, Alexandre Ern, Miguel Angel Fernández

\section{To cite this version:}

Erik Burman, Alexandre Ern, Miguel Angel Fernández. Fractional-step methods and finite elements with symmetric stabilization for the transient Oseen problem. ESAIM: Mathematical Modelling and Numerical Analysis, 2017, 51 (2), pp.487-507. 10.1051/m2an/2016028 . hal-01218328

\section{HAL Id: hal-01218328 \\ https://hal.inria.fr/hal-01218328}

Submitted on 20 Oct 2015

HAL is a multi-disciplinary open access archive for the deposit and dissemination of scientific research documents, whether they are published or not. The documents may come from teaching and research institutions in France or abroad, or from public or private research centers.
L'archive ouverte pluridisciplinaire HAL, est destinée au dépôt et à la diffusion de documents scientifiques de niveau recherche, publiés ou non, émanant des établissements d'enseignement et de recherche français ou étrangers, des laboratoires publics ou privés. 
Fractional-step methods and finite elements with symmetric stabilization for the transient Oseen problem

Erik Burman, Alexandre Ern, Miguel A. Fernández

RESEARCH

REPORT

$\mathbf{N}^{\circ} 8799$

October 2015

Project-Team REO 



\title{
inzián
}

\section{Fractional-step methods and finite elements with symmetric stabilization for the transient Oseen problem}

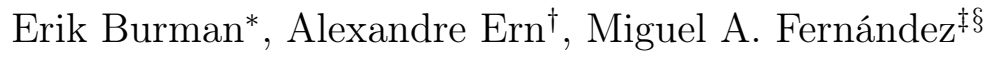 \\ Project-Team REO \\ Research Report n 8799 - October 2015 - 24 pages
}

\begin{abstract}
This paper deals with the spatial and time discretization of the transient Oseen equations. Finite elements with symmetric stabilization in space are combined with several timestepping schemes (monolithic and fractional-step). Quasi-optimal (in space) and optimal (in time) error estimates are established for smooth solutions in all flow regimes. We first analyze monolithic time discretizations using the Backward Differentation Formulas of order 1 and 2 (BDF1 and BDF2). We derive a new estimate on the time-average of the pressure error featuring the same robustness with respect to the Reynolds number as the velocity estimate. Then, we analyze fractional-step pressure-projection methods using BDF1. The stabilization of velocities and pressures can be treated either implicitly or explicitly. Numerical results illustrate the main theoretical findings.
\end{abstract}

Key-words: Oseen equations, stabilized finite elements, fractional-step methods, pressurecorrection methods, error estimates, high Reynolds number.

* Department of Mathematics, Mantell Building, University of Sussex, Brighton, BN1 9RF United Kingdom

† Université Paris-Est, CERMICS (ENPC), 77455 Marne la Vallée Cedex 2, France

‡ Inria, REO project-team, Rocquencourt - B.P. 105, F-78153 Le Chesnay cedex, France

$\S$ Sorbonne Universités, UPMC Univ. Paris 6, Lab. JLL, 4 Place Jussieu, 75252 Paris cedex 05, France

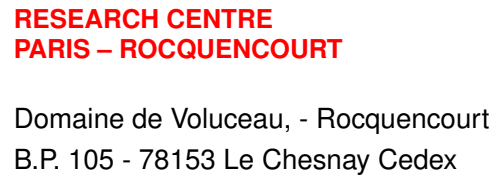




\section{Méthodes à pas fractionnaire et éléments finis avec stabilisation symétrique pour le problème d'Oseen transitoire}

Résumé : Cet article est consacré à la discrétisation spatiale et temporelle des équations d'Oseen transitoires. Des méthodes d'éléments finis stabilisées symétriques en espace sont combinées avec plusieurs schémas de discrétisation en temps (monolithiques et à pas fractionnaire). Des estimations d'erreur quasi-optimales (en espace) et optimales (en temps) sont établies pour des solutions régulières dans tous les régimes de l'écoulement. Nous considérons d'abord des discrétisations de temps monolithiques avec des formules BDF d'ordre 1 et 2 (BDF1 et BDF2). Nous obtenons une nouvelle estimation de la moyenne en temps de l'erreur en pression avec la même robustesse (par rapport au nombre de Reynolds) que l'estimation de l'erreur en vitesse. Nous analysons ensuite des méthodes à pas fractionnaire de type projection de la pression en utilisant du BDF1. La stabilisation de la vitesse et de la pression peuvent être traités implicitement ou explicitement. Les résultats numériques illustrent les principales conclusions théoriques.

Mots-clés : Équations d'Oseen, éléments finis stabilisés, méthodes à pas fractionnaire, méthodes de correction de la pression, estimations d'erreur, nombre de Reynolds élevé. 


\section{Introduction}

The computation of Navier-Stokes flows at high Reynolds number is an important challenge in scientific computation. Many space discretization methods rely crucially on the presence of non-negligible viscous dissipation and therefore lack robustness when the mesh Reynolds number $\frac{|\beta| h}{\mu}$ ( $\beta$ is the flow velocity, $h$ the mesh size, and $\mu$ the viscosity parameter) is much larger than one. In this regime, the standard Galerkin formulation is known to be unstable even in the linear case, and these instabilities tend to be amplified by nonlinearities. To counter such instabilities, one may resort to stabilization techniques, such as SUPG [17], discontinuous Galerkin [18, or $H^{1}$-conforming finite elements with symmetric stabilization in various flavors [12, 8, 6, 1]. The effect of stabilization is on the one hand to improve the convergence to smooth solutions. On the other hand, for rough solutions, stabilization limits the propagation of perturbations generated in the vicinity of sharp gradients; stabilization also turns out to promote the Gibbs phenomenon when approximating rough solutions to conservation laws, and this effect can be tempered by some nonlinear weighting mechanism [10].

In this paper, we are interested in combining stabilized finite elements for space discretization with time discretization schemes for fluid flow problems in the high Reynolds number regime. The importance of stabilization in the high Reynolds number regime for fractional-step methods was illustrated numerically in 13 for Navier-Stokes flows, showing that the pressure-projection method can fail to converge in the high Reynolds number regime unless some stabilization is applied. Our goal here is to provide some theoretical background analyzing this fact. For simplicity, we focus on the linearized version of the Navier-Stokes equations known as the Oseen equations, and we prove that stabilized finite element methods also improve the convergence rate to smooth solutions when using a fractional-step pressure-projection scheme for time discretization. Let $\Omega$ be a bounded polyhedron in $\mathbb{R}^{d}, d \in\{2,3\}$, with boundary $\partial \Omega$ and outward normal $\nu$, and let $t_{\mathrm{F}}$ be a finite positive time. We consider the Oseen equations posed in the space-time cylinder $Q:=\Omega \times\left(0, t_{\mathrm{F}}\right)$,

$$
\begin{aligned}
\partial_{t} u+\beta \cdot \nabla u-\mu \Delta u+\nabla p & =f, \\
\nabla \cdot u & =0,
\end{aligned}
$$

where the unknowns are the velocity field $u$ and the pressure $p$, while the data are the advection velocity $\beta$, the viscosity $\mu$, and the body force $f$. We assume that the velocity field $\beta$ is Lipschitz (with Lipschitz constant denoted by $L_{\beta}$ ) and divergence-free, that $\mu$ is a positive real number, and that $f \in L^{2}\left(0, t_{\mathrm{F}} ; L^{2}(\Omega)^{d}\right)$. The Oseen equations are supplemented with a homogeneous Dirichlet boundary condition on the velocity and an initial condition on the velocity of the form $\left.u\right|_{t=0}=u_{0}$, with $u_{0} \in H_{0}^{1}(\Omega)^{d}$ and $\nabla \cdot u_{0}=0$.

The quasi-optimal approximation of smooth solutions to the stationary Oseen equations using finite elements with symmetric stabilization in various flavors has been investigated in [2]. In the unstationary case, the literature on projection methods for the Navier-Stokes equations is very rich, starting with the pioneering work by Chorin and Temam, Yosida and more recent work (see, e.g., [21, 14, 9] and references therein). Nevertheless, a complete analysis including space discretization using stabilized finite elements is, to our knowledge, not yet available, even in the linearized case of the unstationary Oseen equations. In the present work, we provide such an analysis. We focus on stabilization using the continuous interior penalty finite element method analyzed in 4 for the stationary Oseen equations. Stabilization concerns the advective derivative, the pressure-velocity coupling, and the velocity divergence. Moreover, the homogeneous Dirichlet boundary condition on the velocity is enforced weakly using the classical boundary penalty method of Nitsche [20]. 
This paper is organized as follows. In Section 2 we present the discrete setting. In Section 3 , we analyze monolithic time discretizations, including both first- and second-order Backward Differentiation (BDF) schemes. The main result is Theorem 3.1. While the velocity estimate follows using standard arguments based on [5, the pressure estimate, inspired by the recent asymptotic analysis of [7, is, to our knowledge, new. The idea is to bound the time-average of the pressure error, so as to achieve the same type of robustness with respect to the Reynolds number as for the velocity estimate. Note that stabilization plays a role in achieving this result. In Section 4, we analyze the fractional-step time discretization using pressure projection, focusing on the first-order BDF scheme. The main result is Theorem 4.1 providing (quasi-optimal in space and optimal in time) error estimates that are independent of the viscosity (but not of high-order Sobolev norms of the exact solution). Moreover, Corollary 4.1 shows that it is possible to treat velocity and/or pressure stabilization either implicitly or explicitly in time, up to some modifications of the CFL condition on the time step. From the point of view of computation, it can be advantageous to treat the stabilization explicitly. In particular, this eliminates the need for the construction of a system matrix with a nonstandard stencil. Finally, in Section 5 , we present numerical results illustrating the theoretical analysis.

\section{The discrete setting}

Let $L:=L^{2}(\Omega)$ and $L_{*}:=\left\{q \in L ; \int_{\Omega} q=0\right\}$. For a subset $S \subset \Omega$, we denote by $(\cdot, \cdot)_{L, S}$ and $\|\cdot\|_{L, S}$ respectively the standard inner product and norm of $L^{2}(S)$, with the convention that the index $S$ is omitted if $S=\Omega$.

\subsection{Space semi-discrete Oseen equations}

Let $\left\{\mathcal{T}_{h}\right\}_{h>0}$ be a family of affine, simplicial meshes of $\Omega$. We assume that the meshes are kept fixed in time and, for simplicity, the family $\left\{\mathcal{T}_{h}\right\}_{h>0}$ is supposed to be quasi-uniform. Mesh faces are collected in the set $\mathcal{F}_{h}$ which is split into the set of interior faces, $\mathcal{F}_{h}^{\text {int }}$, and of boundary faces, $\mathcal{F}_{h}^{\text {ext }}$. For a smooth enough function $v$ that is possibly double-valued at $F \in \mathcal{F}_{h}^{\text {int }}$ with $F=\partial T^{-} \cap \partial T^{+}$, we define its jump at $F$ as $\llbracket v \rrbracket:=\left.v\right|_{T^{-}}-\left.v\right|_{T^{+}}$, and we fix the unit normal vector to $F$, denoted by $\nu_{F}$, as pointing from $T^{-}$to $T^{+}$. The arbitrariness in the sign of $\llbracket v \rrbracket$ is irrelevant in what follows.

We consider continuous finite elements with equal-order to discretize in space the velocity and the pressure. Let $k \geq 1$ be an integer and set

$$
M_{h}:=\left\{m_{h} \in C^{0}(\bar{\Omega}) ; \forall T \in \mathcal{T}_{h},\left.m_{h}\right|_{T} \in \mathbb{P}_{k}(T)\right\},
$$

with $\mathbb{P}_{k}(T)$ spanned by the restriction to $T$ of polynomials of total degree $\leq k$. Set

$$
V_{h}:=\left[M_{h}\right]^{d}, \quad P_{h}=M_{h} \cap L_{*},
$$

and observe that the boundary condition on the velocity is to be enforced weakly. We also need the extended space

$$
\widetilde{V}_{h}:=V_{h}+\nabla P_{h}
$$

To express the divergence-free constraint at the discrete level, we consider the discrete operator $B_{h}: V_{h} \rightarrow P_{h}$ such that for all $\left(v_{h}, q_{h}\right) \in V_{h} \times P_{h}$,

$$
\left(B_{h} v_{h}, q_{h}\right)_{L}:=-\left(\nabla \cdot v_{h}, q_{h}\right)_{L}+\left(\nu \cdot v_{h}, q_{h}\right)_{L, \partial \Omega}
$$


and its transpose $B_{h}^{\mathrm{T}}: P_{h} \rightarrow V_{h}$. As motivated in [15], we also consider the extension of $B_{h}$ to $\widetilde{V}_{h}$, namely $C_{h}: \widetilde{V}_{h} \rightarrow P_{h}$ such that for all $\left(\widetilde{v}_{h}, q_{h}\right) \in \widetilde{V}_{h} \times P_{h}$,

$$
\left(C_{h} \widetilde{v}_{h}, q_{h}\right)_{L}:=\left(\widetilde{v}_{h}, \nabla q_{h}\right)_{L} .
$$

Integration by parts yields $B_{h}=C_{h} i_{h}$ where $i_{h}$ is the canonical injection of $V_{h}$ into $\widetilde{V}_{h}$. Moreover, the transpose $C_{h}^{\mathrm{T}}: P_{h} \rightarrow \widetilde{V}_{h}$ is the restriction of the gradient operator to $P_{h}$, and we infer that $B_{h}^{\mathrm{T}}=i_{h}^{\mathrm{T}} C_{h}^{\mathrm{T}}$ where $i_{h}^{\mathrm{T}}$ coincides with the (restriction to $\widetilde{V}_{h}$ of the) $L$-orthogonal projection onto $V_{h}$, henceforth denoted by $\pi_{h}$. To alleviate the notation in what follows, we omit the operator $i_{h}$. We extend the domains of $B_{h}, C_{h}$, and their transposes to smooth functions by setting $\left(B_{h} v, q_{h}\right)_{L}:=-\left(\nabla \cdot v, q_{h}\right)_{L}+\left(\nu \cdot v, q_{h}\right)_{L, \partial \Omega}$ and $\left(C_{h} v, q_{h}\right)_{L}:=\left(v, \nabla q_{h}\right)_{L}$ for all $q_{h} \in P_{h}$ and all $v \in\left[H^{1}(\Omega)\right]^{d}$, and $C_{h}^{\mathrm{T}} q=\nabla q$ for all $q \in H^{1}(\Omega)$, while $B_{h}^{\mathrm{T}} q=i_{h}^{\mathrm{T}} \nabla q$.

To discretize the convection-diffusion operator in 1a), we consider the continuous interior penalty finite element method analyzed in 4 for the stationary Oseen equations. We introduce the discrete operator $A_{h}: V_{h} \rightarrow V_{h}$ such that, for all $\left(v_{h}, w_{h}\right) \in V_{h} \times V_{h}$,

$$
\begin{aligned}
\left(A_{h} v_{h}, w_{h}\right)_{L}:= & \left(\beta \cdot \nabla v_{h}, w_{h}\right)_{L}+\sum_{F \in \mathcal{F}_{h}^{\text {ext }}}\left(\left(\beta \cdot \nu_{F}\right)^{\ominus} v_{h}, w_{h}\right)_{L, F} \\
& +\left(\mu \nabla v_{h}, \nabla w_{h}\right)_{L}-\left(\mu\left(\nu \cdot \nabla v_{h}\right), w_{h}\right)_{L, \partial \Omega}-\left(v_{h}, \mu\left(\nu \cdot \nabla w_{h}\right)\right)_{L, \partial \Omega} \\
& +\sum_{F \in \mathcal{F}_{h}^{\text {ext }}} \gamma_{1} h_{F}^{-1}\left(\mu v_{h}, w_{h}\right)_{L, F},
\end{aligned}
$$

where for a real number $x, x^{\ominus}=\frac{1}{2}(|x|-x)$ denotes its negative part $\left(x^{\oplus}=\frac{1}{2}(|x|+x)\right.$ denotes its positive part), and where $\gamma_{1}$ is a user-dependent positive parameter related to the boundary penalty method. Furthermore, we consider the stabilization operators $S_{h}^{u}: V_{h} \rightarrow V_{h}$ and $S_{h}^{p}$ : $P_{h} \rightarrow P_{h}$ such that for all $\left(v_{h}, w_{h}\right) \in V_{h} \times V_{h}$ and $\left(q_{h}, r_{h}\right) \in P_{h} \times P_{h}$,

$$
\begin{aligned}
\left(S_{h}^{u} v_{h}, w_{h}\right)_{L}:= & \sum_{F \in \mathcal{F}_{h}^{\text {int }}} \gamma_{2} \xi_{F} \beta_{\nu, F} h_{F}^{2}\left(\llbracket \nu_{F} \cdot \nabla v_{h} \rrbracket, \llbracket \nu_{F} \cdot \nabla w_{h} \rrbracket\right)_{L, F} \\
& +\sum_{F \in \mathcal{F}_{h}^{\text {int }}} \gamma_{3} \xi_{F} \beta_{F} h_{F}^{2}\left(\llbracket \nabla \cdot v_{h} \rrbracket, \llbracket \nabla \cdot w_{h} \rrbracket\right)_{L, F} \\
& +\sum_{F \in \mathcal{F}_{h}^{\text {ext }}} \gamma_{3} \beta_{F}\left(\nu_{F} \cdot v_{h}, \nu_{F} \cdot w_{h}\right)_{L, F} \\
\left(S_{h}^{p} q_{h}, r_{h}\right)_{L}:= & \sum_{F \in \mathcal{F}_{h}^{\text {int }}} \gamma_{4} \xi_{F} \beta_{F}^{-1} h_{F}^{2}\left(\llbracket \nabla q_{h} \rrbracket, \llbracket \nabla r_{h} \rrbracket\right)_{L, F},
\end{aligned}
$$

with local velocities $\beta_{\nu, F}:=\left\|\beta \cdot \nu_{F}\right\|_{L^{\infty}(F)}$ and $\beta_{F}:=\|\beta\|_{\left[L^{\infty}(F)\right]^{d}}$, user-dependent positive parameters $\gamma_{2}, \gamma_{3}$, and $\gamma_{4}$, and cut-off function $\xi_{F}=\min \left(1, \operatorname{Re}_{F}\right)$ with local face Reynolds number $\operatorname{Re}_{F}:=\frac{h_{F} \beta_{F}}{\mu}$ (note that in $8 \mathrm{~b}, \xi_{F} \beta_{F}^{-1}$ is bounded by $\mu^{-1} h_{F}$ if $\beta$ vanishes on $F$ ). The aim of the stabilization operators is to stabilize the advection operator (sum with $\gamma_{2}$ ), achieve additional control on the incompressibility condition (sums with $\gamma_{3}$ ), and ensure inf-sup stability for the pressure-velocity coupling (sum with $\gamma_{4}$ ). The domain of the discrete operators $A_{h}, S_{h}^{u}$, and $S_{h}^{p}$ can be extended to smooth functions by setting $A_{h} v=\pi_{h}(\beta \cdot \nabla v-\mu \Delta v), S_{h}^{u} v=0$ for all $v \in\left[H^{2}(\Omega) \cap H_{0}^{1}(\Omega)\right]^{d}$, and $S_{h}^{p} q=0$ for all $q \in H^{2}(\Omega)$.

The space semi-discrete Oseen equations take the following form: Find $u_{h} \in C^{1}\left(\left[0, t_{\mathrm{F}}\right] ; V_{h}\right)$ and $p_{h} \in C^{0}\left(\left[0, t_{\mathrm{F}}\right] ; P_{h}\right)$ such that, for all $t \in\left(0, t_{\mathrm{F}}\right)$,

$$
\begin{aligned}
\partial_{t} u_{h}+A_{h} u_{h}+S_{h}^{u} u_{h}+B_{h}^{\mathrm{T}} p_{h} & =f_{h} & & \left(\text { in } V_{h}\right), \\
-B_{h} u_{h}+S_{h}^{p} p_{h} & =0 & & \left(\text { in } P_{h}\right),
\end{aligned}
$$

$\mathrm{RR} \mathrm{n}^{\circ} 8799$ 
where $f_{h}:=\pi_{h} f$. This problem was analyzed in [5].

\subsection{Analysis tools}

We consider the following norm on $V_{h}+\left[H^{2}(\Omega)\right]^{d}$ :

$$
\|v\|_{\mu, \beta}:=\mu^{\frac{1}{2}}\left(\|\nabla v\|_{L}+h^{-\frac{1}{2}}\|v\|_{L, \partial \Omega}+h^{\frac{1}{2}}\|\nu \cdot \nabla v\|_{L, \partial \Omega}\right)+\left\||\beta \cdot \nu|^{\frac{1}{2}} v\right\|_{L, \partial \Omega},
$$

together with the following semi-norms on $V_{h}+\left[H^{2}(\Omega)\right]^{d}$ and $P_{h}+H^{2}(\Omega)$, respectively,

$$
|v|_{S^{u}}:=\left(S_{h}^{u} v, v\right)_{L}^{\frac{1}{2}}, \quad|q|_{S^{p}}:=\left(S_{h}^{p} q, q\right)_{L}^{\frac{1}{2}} .
$$

It is well-known that for $\gamma_{1}$ large enough, using integration by parts and discrete trace inequalities, the following holds for all $v_{h} \in V_{h}$ :

$$
\left(A_{h} v_{h}, v_{h}\right)_{L} \gtrsim\left\|v_{h}\right\|_{\mu, \beta}^{2} .
$$

The above assumption on $\gamma_{1}$ is implicitly made in what follows. Here and in what follows, we abbreviate $A \lesssim B$ the inequality $A \leq c B$ for positive real numbers $A$ and $B$, where the value of $c$ can change at each occurrence while being independent of the mesh size and the physical parameters $\beta, \mu$ and $t_{\mathrm{F}}$ ( $c$ can depend on the polynomial degree $k$ and the stabilization constants $\left.\gamma_{i}, i=1, \ldots, 4\right)$.

Lemma 2.1 (Boundedness on orthogonal subscales). Let $v \in V_{h}+\left[H^{2}(\Omega)\right]^{d}$ and let $q \in M_{h}+$ $H^{2}(\Omega)$. Assume that $\left(v, w_{h}\right)_{L}=0$ for all $w_{h} \in V_{h}$ and that $\left(q, r_{h}\right)_{L}=0$ for all $r_{h} \in M_{h}$ (functions satisfying such properties are called orthogonal subscales). Let $c(\beta, h):=\max _{F \in \mathcal{F}_{h}^{\mathrm{int}}}\left(\xi_{F}^{-1} \beta_{F}\right)$ and $\check{c}(\beta, h):=\max _{F \in \mathcal{F}_{h}}\left(\xi_{F} \beta_{F}^{-1}\right)$. Then, for all $q_{h} \in P_{h}$ and all $v_{h} \in V_{h}$, the following holds:

$$
\begin{aligned}
\left(C_{h} v, q_{h}\right)_{L} & \lesssim c(\beta, h)^{\frac{1}{2}} h^{-\frac{1}{2}}\|v\|_{L}\left|q_{h}\right|_{S^{p}}, \\
\left(B_{h}^{\mathrm{T}} q, v_{h}\right)_{L} & \lesssim \check{c}(\beta, h)^{\frac{1}{2}}\left(h^{-\frac{1}{2}}\|q\|_{L}+\|q\|_{L, \partial \Omega}\right)\left(\left|v_{h}\right|_{S^{u}}+\left\|v_{h}\right\|_{\mu, \beta}\right), \\
\left(A_{h} v, v_{h}\right)_{L} & \lesssim c(\beta, h)^{\frac{1}{2}} h^{-\frac{1}{2}}\|v\|_{L}\left|v_{h}\right|_{S^{u}}+L_{\beta}\|v\|_{L}\left\|v_{h}\right\|_{L}+\|v\|_{\mu, \beta}\left\|v_{h}\right\|_{\mu, \beta} .
\end{aligned}
$$

Proof. We only sketch the proof and refer to [4 for further insight. The bound 13a results from

$$
\left(C_{h} v, q_{h}\right)_{L}=\left(v, \nabla q_{h}\right)_{L}=\inf _{y_{h} \in V_{h}}\left(v, \nabla q_{h}-y_{h}\right)_{L} \leq\|v\|_{L} \inf _{y_{h} \in V_{h}}\left\|\nabla q_{h}-y_{h}\right\|_{L} .
$$

Taking for $y_{h}$ a quasi-interpolate of $\nabla q_{h}$ in $V_{h}$ based on averaging and proceeding as in 44, we infer that $\inf _{y_{h} \in V_{h}}\left\|\nabla q_{h}-y_{h}\right\|_{L} \lesssim c(\beta, h)^{\frac{1}{2}} h^{-\frac{1}{2}}\left|q_{h}\right|_{S^{p}}$. To prove $\left.113 \mathrm{~b}\right)$, we first observe that

$$
\left(B_{h}^{\mathrm{T}} q, v_{h}\right)_{L}=\inf _{r_{h} \in M_{h}}\left(q, \nabla \cdot v_{h}-r_{h}\right)_{L}+\left(q, \nu \cdot v_{h}\right)_{L, \partial \Omega} .
$$

The first term in the right-hand side, say $\mathfrak{T}_{1}$, can be bounded by

$$
\left|\mathfrak{T}_{1}\right| \lesssim h^{-\frac{1}{2}}\|q\|_{L}\left(\max _{F \in \mathcal{F}_{h}^{\text {int }}} \min \left(\xi_{F}^{-1} \beta_{F}^{-1}, h_{F} \mu^{-1}\right)\right)^{\frac{1}{2}}\left(\left|v_{h}\right|_{S^{u}}+\left\|v_{h}\right\|_{\mu, \beta}\right) .
$$

Then, observing that $\min \left(\xi_{F}^{-1} \beta_{F}^{-1}, h_{F} \mu^{-1}\right)=\min \left(\beta_{F}^{-1}, h_{F} \mu^{-1}\right)=\xi_{F} \beta_{F}^{-1}$ and proceeding similarly for the boundary term, we obtain $113 \mathrm{~b}$. Finally, for $113 \mathrm{c})$, denoting by $\bar{\beta}$ the element-wise average of the velocity field $\beta$, we infer that

$$
\left(A_{h} v, w_{h}\right)_{L} \lesssim \inf _{y_{h} \in V_{h}}\left\{-\left(v, \bar{\beta} \cdot \nabla w_{h}-y_{h}\right)_{L}\right\}+\left(v,(\bar{\beta}-\beta) \cdot \nabla w_{h}\right)_{L}+\|v\|_{\mu, \beta}\left\|w_{h}\right\|_{\mu, \beta},
$$

using Cauchy-Schwarz inequalities on the diffusive part and the boundary terms. For the convective terms, we conclude as above for the first term in the right-hand side (note that $\beta_{\nu, F} \leq \beta_{F}$ ), the Lipschitz property of $\beta$, and inverse inequalities. 
For the proof of the following approximation results, we refer to [4]. For simplicity, we assume that the functions to approximate are smooth enough. We also use $\pi_{h}$ to denote the $L$-orthogonal projection onto $M_{h}$ as well as that onto $V_{h}$.

Lemma 2.2 (Approximation). Let $k \geq 1$ be the polynomial degree. Assume that $v \in\left[H^{k+1}(\Omega)\right]^{d}$ and $q \in H^{k+1}(\Omega)$. Set $\beta_{\Omega}:=\|\beta\|_{\left[L^{\infty}(\Omega)\right]^{d}}$. Then, the following holds:

$$
\begin{aligned}
& \left\|v-\pi_{h} v\right\|_{L} \lesssim h^{k+1}|v|_{\left[H^{k+1}(\Omega)\right]^{d}}, \\
& \left\|q-\pi_{h} q\right\|_{L}+h^{\frac{1}{2}}\left\|q-\pi_{h} q\right\|_{L, \partial \Omega} \lesssim h^{k+1}|q|_{H^{k+1}(\Omega)}, \\
& \left\|v-\pi_{h} v\right\|_{\mu, \beta} \lesssim c(\beta, h)^{\frac{1}{2}} h^{k+\frac{1}{2}}|v|_{\left[H^{k+1}(\Omega)\right]^{d}}, \\
& \left|v-\pi_{h} v\right|_{S^{u}} \lesssim \check{c}(\beta, h)^{\frac{1}{2}} \beta_{\Omega} h^{k+\frac{1}{2}}|v|_{\left.H^{k+1}(\Omega)\right]^{d}}, \\
& \left|q-\pi_{h} q\right|_{S^{p}} \lesssim \check{c}(\beta, h)^{\frac{1}{2}} h^{k+\frac{1}{2}}|q|_{H^{k+1}(\Omega)} .
\end{aligned}
$$

\section{Monolithic time discretization}

Let $\tau$ be the time step, taken to be constant for simplicity and such that $N \tau=t_{\mathrm{F}}$. We define the Courant number as

$$
\mathrm{Co}:=\frac{\beta_{\Omega} \tau}{h}
$$

In what follows, for $0 \leq n \leq N$, a superscript $n$ indicates the values of a function at the discrete time $n \tau$, e.g., $u^{n}=u\left(t^{n}\right)$ and $p^{n}=p\left(t^{n}\right)$. In what follows, we assume at least that $u^{n} \in\left[H^{2}(\Omega)\right]^{d}$ and $p^{n} \in H^{2}(\Omega)$ for all $n \geq 1$.

In this section, we consider a BDF1 $(l=1)$ or BDF2 $(l=2)$ monolithic time discretization of the space semi-discrete problem $(9 a)-(9 b)$. The fully discrete scheme takes the following form: For all $n \geq l-1$, find $u_{h}^{n+1} \in V_{h}$ and $p_{h}^{n+1} \in P_{h}$ such that

$$
\begin{aligned}
\partial_{\tau}^{l} u_{h}^{n+1}+A_{h} u_{h}^{n+1}+ & S_{h}^{u} u_{h}^{n+1}+B_{h}^{\mathrm{T}} p_{h}^{n+1}=f_{h}^{n+1} & & \left(\text { in } V_{h}\right), \\
-B_{h} u_{h}^{n+1}+S_{h}^{p} p_{h}^{n+1} & =0 & & \left(\text { in } P_{h}\right),
\end{aligned}
$$

where $f_{h}:=\pi_{h} f, \partial_{\tau}^{1} u_{h}^{n+1}=\frac{1}{\tau}\left(u_{h}^{n+1}-u_{h}^{n}\right)$, and $\partial_{\tau}^{2} u_{h}^{n+1}=\frac{1}{\tau}\left(u_{h}^{n+1}-4 u_{h}^{n}+3 u_{h}^{n-1}\right)$.

We now derive error estimates for the velocity and the pressure. Define the velocity error $e^{n}:=u^{n}-u_{h}^{n}$ and the pressure error $\eta^{n}:=p^{n}-p_{h}^{n}$. It is readily seen that the equations governing the velocity and pressure errors read as follows:

$$
\begin{aligned}
\pi_{h} \partial_{\tau}^{l} e^{n+1}+A_{h} e^{n+1}+S_{h}^{u} e^{n+1}+B_{h}^{\mathrm{T}} \eta^{n+1} & =\pi_{h} \Psi_{l}^{n+1} & & \left(\text { in } V_{h}\right), \\
-B_{h} e^{n+1}+S_{h}^{p} \eta^{n+1} & =0 & & \left(\text { in } P_{h}\right),
\end{aligned}
$$

where $\Psi_{l}^{n}:=\partial_{\tau}^{l} u^{n}-\left(\partial_{t} u\right)^{n}$ for all $n \geq 1$.

In what follows, we consider the discrete $L^{2}$-in-time (at the time nodes) norm of a space-time function $z$ that we denote $\|z\|_{\ell^{2}\left(0, t_{\mathrm{F}} ; Z\right)}^{2}:=\tau \sum_{n=1}^{N}\left\|z\left(t^{n}\right)\right\|_{Z}^{2}$ where $Z$ is some space of functions in space. We define the time-averaged pressure error $\bar{\eta}^{N}:=N^{-1} \sum_{n=l}^{N} \eta^{n}$. To avoid technicalities with the initialization of the scheme, we neglect the error for $n \leq l-1$. To simplify the pressure estimate, we assume that $\max \left(\mu, \beta_{F} h_{F}\right) \leq 1$ for all $F \in \mathcal{F}_{h}$, so that $c(\beta, h) h \leq 1$, that $\beta_{\Omega} \leq 1$, and that $t_{\mathrm{F}} \geq 1$. 
Theorem 3.1 (Error estimates). Assume that $u\left(t^{n}\right) \in\left[H^{k+1}(\Omega)\right]^{d}$ and $p\left(t^{n}\right) \in H^{k+1}(\Omega)$ for all $n \geq l$, and $\partial_{t}^{l+1} u \in L^{2}(Q)$. Then, the following estimates hold:

$$
\begin{aligned}
\left\|e^{N}\right\|_{L} & +\left(\sum_{n \geq l} \tau\left(\left\|e^{n}\right\|_{\mu, \beta}^{2}+\left|e^{n}\right|_{S^{u}}^{2}+\left|\eta^{n}\right|_{S^{p}}^{2}\right)\right)^{\frac{1}{2}} \\
\lesssim & \left(c(\beta, h)^{\frac{1}{2}}+\check{c}(\beta, h)^{\frac{1}{2}} \beta_{\Omega}+t_{\mathrm{F}}^{\frac{1}{2}} L_{\beta} h^{\frac{1}{2}}\right) h^{k+\frac{1}{2}}\|u\|_{\ell^{2}\left(0, t_{F} ; H^{k+1}(\Omega)\right)} \\
& +\check{c}(\beta, h)^{\frac{1}{2}} h^{k+\frac{1}{2}}\|p\|_{\ell^{2}\left(0, t_{F} ; H^{k+1}(\Omega)\right)}+t_{\mathrm{F}}^{\frac{1}{2}} \tau^{l}\left\|\partial_{t}^{l+1} u\right\|_{L, Q}, \\
\left\|\bar{\eta}^{N}\right\|_{L} \lesssim & h^{k+1}\left\|\bar{p}^{N}\right\|_{H^{k+1}(\Omega)} \\
& +t_{\mathrm{F}}^{-\frac{1}{2}}\left(c(\beta, h)^{\frac{1}{2}}+\check{c}(\beta, h)^{\frac{1}{2}} \beta_{\Omega}+t_{\mathrm{F}}^{\frac{1}{2}} L_{\beta} h^{\frac{1}{2}}\right) h^{k+\frac{1}{2}}\|u\|_{\ell^{2}\left(0, t_{F} ; H^{k+1}(\Omega)\right)} \\
& +t_{\mathrm{F}}^{-\frac{1}{2}} \check{c}(\beta, h)^{\frac{1}{2}} h^{k+\frac{1}{2}}\|p\|_{\ell^{2}\left(0, t_{F} ; H^{k+1}(\Omega)\right)}+t_{\mathrm{F}}^{-\frac{1}{2}} \tau^{l}\left\|\partial_{t}^{l+1} u\right\|_{L, Q} .
\end{aligned}
$$

Proof. The estimate (18a) can be derived using the arguments of [5], the boundedness estimates from Lemma 2.1, the approximation results from Lemma 2.2, and the standard truncation error estimates for the discrete time derivative. We detail here only the proof of $\sqrt{18 \mathrm{~b}})$. Let $\bar{v}_{p} \in$ $\left[H_{0}^{1}(\Omega)\right]^{d}$ be such that

$$
\nabla \cdot \bar{v}_{p}=\bar{\eta}^{N}, \quad\left\|\bar{v}_{p}\right\|_{\left[H^{1}(\Omega)\right]^{d}} \lesssim\left\|\bar{\eta}^{N}\right\|_{L} .
$$

Then, using $y_{h}^{n}=\pi_{h} p^{n}-p_{h}^{n}$, and letting $\bar{y}_{h}^{N}$ and $\bar{p}^{N}$ be the discrete time averages of the functions $y_{h}^{n}$ and $p^{n}$, we infer that

$$
\begin{aligned}
\left\|\bar{\eta}^{N}\right\|_{L}^{2} & =\left(\bar{\eta}^{N}, \nabla \cdot \bar{v}_{p}\right)_{L}=\left(\bar{\eta}^{N}, \nabla \cdot\left(\bar{v}_{p}-\pi_{h} \bar{v}_{p}\right)\right)_{L}+\left(\bar{\eta}^{N}, \nabla \cdot \pi_{h} \bar{v}_{p}\right)_{L} \\
& =\left(\bar{p}^{N}-\pi_{h} \bar{p}^{N}, \nabla \cdot\left(\bar{v}_{p}-\pi_{h} \bar{v}_{p}\right)\right)_{L}+\left(\bar{y}_{h}^{N}, \nabla \cdot\left(\bar{v}_{p}-\pi_{h} \bar{v}_{p}\right)\right)_{L}+\left(\bar{\eta}^{N}, \nabla \cdot \pi_{h} \bar{v}_{p}\right)_{L} \\
& =-\left(\nabla\left(\bar{p}^{N}-\pi_{h} \bar{p}^{N}\right), \bar{v}_{p}-\pi_{h} \bar{v}_{p}\right)_{L}-\left(\bar{y}_{h}^{N}, C_{h}\left(\bar{v}_{p}-\pi_{h} \bar{v}_{p}\right)\right)_{L}+\left(\bar{\eta}^{N}, \nabla \cdot \pi_{h} \bar{v}_{p}\right)_{L} .
\end{aligned}
$$

Let us denote $\mathfrak{T}_{1,2,3}$ the three terms in the right-hand side. We first observe that

$$
\left|\mathfrak{T}_{1}\right| \lesssim h^{k+1}\left|\bar{p}^{N}\right|_{H^{k+1}(\Omega)}\left\|\bar{\eta}^{N}\right\|_{L}
$$

The second term is bounded using leading to

$$
\left|\mathfrak{T}_{2}\right| \lesssim c(\beta, h)^{\frac{1}{2}} h^{-\frac{1}{2}}\left\|\bar{v}_{p}-\pi_{h} \bar{v}_{p}\right\|_{L}\left|\bar{y}_{h}^{N}\right|_{S^{p}} \lesssim\left|\bar{y}_{h}^{N}\right|_{S_{p}}\left\|\bar{\eta}^{N}\right\|_{L}
$$

where we have used that $c(\beta, h) h \leq 1$ to simplify the estimate. Moreover, using the triangle inequality and a discrete Cauchy-Schwarz inequality in time leads to

$$
\left|\bar{y}_{h}^{N}\right|_{S_{p}} \leq t_{\mathrm{F}}^{-\frac{1}{2}}\left(\sum_{n \geq l} \tau\left|y_{h}^{n}\right|_{S_{p}}^{2}\right)^{\frac{1}{2}}
$$

For the third term, we use $\mathfrak{T}_{3}=-\left(B_{h}^{\mathrm{T}} \bar{\eta}^{N}, \pi_{h} \bar{v}_{p}\right)_{L}$ and we sum 17a over $n$ to infer that

$$
\mathfrak{T}_{3}=\frac{1}{N} \sum_{n \geq l}\left(\partial_{\tau}^{l} e^{n}+A_{h} e^{n}+S_{h}^{u} e^{n}+\Psi_{l}^{n}, \pi_{h} \bar{v}_{p}\right)_{L} .
$$

Since $\pi_{h} \bar{v}_{p}$ does not depend on time, owing to the classical telescoping properties of BDF methods, we infer that $\frac{1}{N} \sum_{n \geq l}\left(\partial_{\tau}^{l} e^{n}, \pi_{h} \bar{v}_{p}\right)_{L} \lesssim t_{\mathrm{F}}^{-1}\left(\left\|e^{N}\right\|_{L}+\left\|e^{N-1}\right\|_{L}\right)\left\|\bar{\eta}^{N}\right\|_{L}$ (the second term is needed 
only for $l=2$ ); note also that $t_{\mathrm{F}}^{-1} \leq t_{\mathrm{F}}^{-\frac{1}{2}}$ owing to the simplifying assumption $t_{\mathrm{F}} \geq 1$. We observe that (using again that $\beta_{F} h_{F} \leq 1$ )

$$
\begin{aligned}
& \left(A_{h} e^{n}, \pi_{h} \bar{v}_{p}\right)_{L} \lesssim\left(\left\|e^{n}\right\|_{\mu, \beta}+\left\|e^{n}\right\|_{L}\right)\left\|\bar{\eta}^{N}\right\|_{L} \\
& \left(S_{h}^{u} e^{n}, \pi_{h} \bar{v}_{p}\right)_{L} \leq\left|e^{n}\right|_{S^{u}}\left|\pi_{h} \bar{v}_{p}\right|_{S^{u}} \lesssim\left|e^{n}\right|_{S^{u}} \max _{F \in \mathcal{F}_{h}}\left(\beta_{F}^{\frac{1}{2}} h_{F}^{\frac{1}{2}}\right)\left\|\bar{v}_{p}\right\|_{\left[H^{1}(\Omega)\right]^{d}} \lesssim\left|e^{n}\right|_{S^{u}}\left\|\bar{\eta}^{N}\right\|_{L}
\end{aligned}
$$

Using a discrete Cauchy-Schwarz inequality in time, we infer that

$$
\frac{1}{N} \sum_{n \geq l}\left(A_{h} e^{n}+S_{h}^{u} e^{n}, \pi_{h} \bar{v}_{p}\right)_{L} \lesssim t_{\mathrm{F}}^{-\frac{1}{2}}\left(\sum_{n \geq l} \tau\left(\left\|e^{n}\right\|_{\mu, \beta}^{2}+\left\|e^{n}\right\|_{L}^{2}+\left|e^{n}\right|_{S^{u}}^{2}\right)\right)^{\frac{1}{2}}\left\|\bar{\eta}^{N}\right\|_{L} .
$$

Moreover, using the classical properties of the time truncation error in BDF methods, we infer that $\left(\Psi_{l}^{n}, \pi_{h} \bar{v}_{p}\right)_{L} \lesssim \tau^{l}\left\|\partial_{t}^{l+1} u\right\|_{L, Q^{n}}\left\|\bar{\eta}^{N}\right\|_{L}$, with $Q^{n}:=\Omega \times\left[t^{n}, t^{n+1}\right]$. The pressure estimate now follows from the velocity estimate.

Remark 3.1 (High Reynolds number). Observe that in the high Reynolds number regime and for smooth $u$ and $p$, we recover the classical velocity estimate of order $h^{k+\frac{1}{2}}+\tau^{l}$, while the estimate on the time-average pressure is of the same order.

\section{Fractional-step time discretization using pressure projec- tion}

Given $u_{h}^{0} \in V_{h}, \widetilde{u}_{h}^{0} \in \widetilde{V}_{h}$, and $p_{h}^{0} \in P_{h}$, the BDF1 projection method consists in solving for all $n \geq 0$,

1. an advection-diffusion problem yielding $u_{h}^{n+1} \in V_{h}$,

$$
\frac{1}{\tau}\left(u_{h}^{n+1}-\pi_{h} \widetilde{u}_{h}^{n}\right)+A_{h} u_{h}^{n+1}+S_{h}^{u}\left(u_{h}^{*}\right)=f_{h}^{n+1}-B_{h}^{\mathrm{T}} p_{h}^{n} \quad\left(\text { in } V_{h}\right),
$$

with the choice $u_{h}^{*}=u_{h}^{n+1}$ for implicit velocity stabilization or $u_{h}^{*}=\pi_{h} \widetilde{u}_{h}^{n}$ or $u_{h}^{*}=u_{h}^{n}$ for explicit velocity stabilization.

2. a Poisson problem with homogeneous Neumann boundary conditions yielding $p_{h}^{n+1} \in P_{h}$ and $\widetilde{u}_{h}^{n+1} \in \widetilde{V}_{h}$,

$$
\begin{aligned}
\frac{1}{\tau}\left(\widetilde{u}_{h}^{n+1}-u_{h}^{n+1}\right)+C_{h}^{\mathrm{T}}\left(p_{h}^{n+1}-p_{h}^{n}\right)+\frac{1}{\tau} \widetilde{S}_{h}^{p} p_{h}^{*}=0 & \left(\text { in } \widetilde{V}_{h}\right), \\
C_{h} \widetilde{u}_{h}^{n+1}=0 & \left(\text { in } M_{h}\right),
\end{aligned}
$$

with $p_{h}^{*}=p_{h}^{n+1}$ (implicit pressure stabilization) or $p_{h}^{*}=p_{h}^{n}$ (explicit pressure stabilization) and where $\widetilde{S}_{h}^{p}: M_{h} \rightarrow \widetilde{V}_{h}$ is such that for all $\left(q_{h}, \widetilde{v}_{h}\right) \in P_{h} \times \widetilde{V}_{h}$,

$$
\left(\widetilde{S}_{h}^{p} q_{h}, \widetilde{v}_{h}\right)_{L}:=\sum_{F \in \mathcal{F}_{h}^{\text {int }}} \gamma_{4} \xi_{F}\|\beta\|_{L^{\infty}(F)^{d}}^{-1} h_{F}^{2}\left(\llbracket \nabla q_{h} \rrbracket, \llbracket \widetilde{v}_{h} \rrbracket\right)_{L, F} .
$$

$R R n^{\circ} 8799$ 
Since $C_{h}^{\mathrm{T}}$ is the restriction of the gradient operator to $P_{h}$, we obtain for all $\left(q_{h}, r_{h}\right) \in P_{h} \times P_{h}$, $\left(\widetilde{S}_{h}^{p} q_{h}, C_{h}^{\mathrm{T}} r_{h}\right)_{L}=\left(S_{h}^{p} q_{h}, r_{h}\right)_{L}$, so that

$$
C_{h} \widetilde{S}_{h}^{p}=S_{h}^{p}
$$

Moreover, since $V_{h}$ is $H^{1}$-conforming, for all $q_{h} \in M_{h}, \widetilde{S}_{h}^{p} q_{h}$ is $L$-orthogonal to $V_{h}$, that is, for all $\left(q_{h}, v_{h}\right) \in P_{h} \times V_{h},\left(\widetilde{S}_{h}^{p} q_{h}, v_{h}\right)_{L}=0$, so that

$$
\pi_{h} \widetilde{S}_{h}^{p}=0 .
$$

Finally, using a discrete trace inequality, we infer that there are $C_{2}, C_{3}$ such that for all $q_{h} \in P_{h}$,

$$
\left\|\widetilde{S}_{h}^{p} q_{h}\right\|_{L} \leq C_{2} \gamma_{4}^{\frac{1}{2}} \check{c}(\beta, h)^{\frac{1}{2}} h^{\frac{1}{2}}\left|q_{h}\right|_{S^{p}} \leq C_{3} \gamma_{4} \check{c}(\beta, h) h^{-\frac{1}{2}}\left\|q_{h}\right\|_{L} .
$$

Applying the projector $\pi_{h}$ to $20 \mathrm{a}$ at step $n$, using $\pi_{h} C_{h}^{\mathrm{T}}=B_{h}^{\mathrm{T}}$ and (23), and combining with 190 yields

$$
\frac{1}{\tau}\left(u_{h}^{n+1}-u_{h}^{n}\right)+A_{h} u_{h}^{n+1}+S_{h}^{u}\left(u_{h}^{*}\right)=f_{h}^{n+1}-B_{h}^{\mathrm{T}}\left(2 p_{h}^{n}-p_{h}^{n-1}\right) \quad\left(\text { in } V_{h}\right) .
$$

Moreover, applying the operator $C_{h}$ to 20a and using (20b) and 220 yields

$$
C_{h} C_{h}^{\mathrm{T}}\left(p_{h}^{n+1}-p_{h}^{n}\right)=\frac{1}{\tau}\left(B_{h} u_{h}^{n+1}-S_{h}^{p} p_{h}^{*}\right) \quad\left(\text { in } P_{h}\right),
$$

since $u_{h}^{n+1} \in V_{h}$ and $C_{h}$ is an extension of $B_{h}$.

\subsection{Stability}

In this section we prove a discrete stability result for the projection scheme 200 . We first prove a generic result without specifying $u^{*}$ and $p^{*}$ and then we detail the different explicit or implicit treatments of the stabilization terms.

Lemma 4.1 (Stability). The following holds:

$$
\begin{aligned}
& \frac{1}{2}\left\|\pi_{h} \widetilde{u}_{h}^{n+1}\right\|_{L}^{2}-\frac{1}{2}\left\|\pi_{h} \widetilde{u}_{h}^{n}\right\|_{L}^{2}+\frac{1}{2}\left\|u_{h}^{n+1}-\pi_{h} \widetilde{u}_{h}^{n}\right\|_{L}^{2}+\tau\left(A_{h} u_{h}^{n+1}, u_{h}^{n+1}\right)_{L}+\tau\left|u_{h}^{n+1}\right|_{S^{u}}^{2} \\
& +\frac{1}{2} \tau^{2}\left\|\nabla p_{h}^{n+1}\right\|_{L}^{2}-\frac{1}{2} \tau^{2}\left\|\nabla p_{h}^{n}\right\|_{L}^{2}+\frac{1}{2} \tau^{2}\left\|\left(I-\pi_{h}\right) \nabla\left(p_{h}^{n+1}-p_{h}^{n}\right)\right\|_{L}^{2}+\tau\left|p_{h}^{n+1}\right|_{S^{p}}^{2} \\
& =\tau\left(f_{h}^{n+1}, u_{h}^{n+1}\right)_{L}+\tau\left(S_{h}^{u}\left(u_{h}^{n+1}-u_{h}^{*}\right), u_{h}^{n+1}\right)_{L}+\tau\left(S_{h}^{p}\left(p_{h}^{n+1}-p_{h}^{*}\right), p_{h}^{n+1}\right)_{L} .
\end{aligned}
$$

Proof. Step 1. Testing 19 with $\tau u_{h}^{n+1}$ yields

$$
\begin{aligned}
\frac{1}{2}\left\|u_{h}^{n+1}\right\|_{L}^{2}-\frac{1}{2}\left\|\pi_{h} \widetilde{u}_{h}^{n}\right\|_{L}^{2}+\frac{1}{2}\left\|u_{h}^{n+1}-\pi_{h} \widetilde{u}_{h}^{n}\right\|_{L}^{2}+\tau\left(A_{h} u_{h}^{n+1}, u_{h}^{n+1}\right)_{L}+\tau\left|u_{h}^{n+1}\right|_{S^{u}}^{2}= \\
\tau\left(f_{h}^{n+1}, u_{h}^{n+1}\right)_{L}+\tau\left(S_{h}^{u}\left(u_{h}^{n+1}-u_{h}^{*}\right), u_{h}^{n+1}\right)_{L}-\tau\left(B_{h}^{\mathrm{T}} p_{h}^{n}, u_{h}^{n+1}\right)_{L} .
\end{aligned}
$$

Step 2. Applying $\pi_{h}$ to $20 \mathrm{a}$ yields

$$
\pi_{h} \widetilde{u}_{h}^{n+1}-u_{h}^{n+1}+\tau B_{h}^{\mathrm{T}}\left(p_{h}^{n+1}-p_{h}^{n}\right)=0 .
$$

Applying $B_{h}$ to this equation, we infer that

$$
B_{h} \pi_{h} \widetilde{u}_{h}^{n+1}-B_{h} u_{h}^{n+1}+\tau B_{h} B_{h}^{\mathrm{T}}\left(p_{h}^{n+1}-p_{h}^{n}\right)=0 .
$$


Applying $C_{h}$ to $20 \mathrm{a}$ and using $20 \mathrm{~b}$ yields

$$
B_{h} u_{h}^{n+1}=\tau C_{h} C_{h}^{\mathrm{T}}\left(p_{h}^{n+1}-p_{h}^{n}\right)+S_{h}^{p} p_{h}^{*},
$$

and subtracting $(31)$ from 30 leads to

$$
B_{h} \pi_{h} \widetilde{u}_{h}^{n+1}=\tau\left(C_{h} C_{h}^{\mathrm{T}}-B_{h} B_{h}^{\mathrm{T}}\right)\left(p_{h}^{n+1}-p_{h}^{n}\right)+S_{h}^{p} p_{h}^{*} .
$$

Testing (31) with $p_{h}^{n}$ and recalling that $C_{h}^{\mathrm{T}}$ is the restriction of the gradient operator to $P_{h}$, we infer that

$$
\tau\left(B_{h}^{\mathrm{T}} p_{h}^{n}, u_{h}^{n+1}\right)_{L}=\frac{1}{2} \tau^{2}\left\|\nabla p_{h}^{n+1}\right\|_{L}^{2}-\frac{1}{2} \tau^{2}\left\|\nabla p_{h}^{n}\right\|_{L}^{2}-\frac{1}{2} \tau^{2}\left\|\nabla\left(p_{h}^{n+1}-p_{h}^{n}\right)\right\|_{L}^{2}+\tau\left(S_{h}^{p} p_{h}^{*}, p_{h}^{n}\right)_{L} .
$$

Step 3. Testing 29 with $\pi_{h} \widetilde{u}_{h}^{n+1}$ and using 32 , we infer that

$$
\begin{aligned}
& \frac{1}{2}\left\|\pi_{h} \widetilde{u}_{h}^{n+1}\right\|_{L}^{2}-\frac{1}{2}\left\|u_{h}^{n+1}\right\|_{L}^{2}+\frac{1}{2} \tau^{2}\left\|\pi_{h} \nabla\left(p_{h}^{n+1}-p_{h}^{n}\right)\right\|_{L}^{2}= \\
& \quad-\tau^{2}\left\|\left(I-\pi_{h}\right) \nabla\left(p_{h}^{n+1}-p_{h}^{n}\right)\right\|_{L}^{2}-\tau\left(S_{h}^{p} p_{h}^{*}, p_{h}^{n+1}-p_{h}^{n}\right)_{L},
\end{aligned}
$$

since $\left\|\pi_{h} \widetilde{u}_{h}^{n+1}-u_{h}^{n+1}\right\|_{L}^{2}=\tau^{2}\left\|\pi_{h} \nabla\left(p_{h}^{n+1}-p_{h}^{n}\right)\right\|_{L}^{2}$.

Step 4. Combining (28) with (33)-(34) and re-arranging terms yields 27).

\subsection{Implicit or explicit of stabilization}

We now apply Lemma 4.1 to the various choices for the arguments of the stabilization operators to show that different options are possible leading to slightly different CFL conditions. To formulate these CFL conditions, we observe using discrete trace and inverse inequalities, that for all $v_{h} \in V_{h}$,

$$
\left|v_{h}\right|_{S^{u}} \leq C_{4} \gamma_{1}^{\frac{1}{2}} \check{c}(\beta, h)^{\frac{1}{2}} \beta_{\Omega}^{\frac{1}{2}} h^{-\frac{1}{2}}\left\|v_{h}\right\|_{L} .
$$

Corollary 4.1. Take $u^{*}=u_{h}^{n+1}$, or $u^{*}=\pi_{h} \tilde{u}_{h}^{n}$ in Lemma 4.1. or for the special case of piecewise linears (i.e., $k=1$ ) take $u^{*}=u_{h}^{n}$. For the pressure stabilization, take $p^{*}=p_{h}^{n+1}$ or $p^{*}=p_{h}^{n}$. Then, the following holds:

$$
\begin{aligned}
\left\|\pi_{h} \widetilde{u}_{h}^{N}\right\|_{L}^{2} & +\tau \sum_{n=0}^{N-1}\left(\left\|u_{h}^{n+1}\right\|_{\mu, \beta}^{2}+\left|u_{h}^{n+1}\right|_{S^{u}}^{2}\right)+\tau^{2}\left\|\nabla p_{h}^{N}\right\|_{L}^{2}+\tau \sum_{n=0}^{N-1}\left|p_{h}^{n+1}\right|_{S^{p}}^{2} \\
& \lesssim \sum_{n=0}^{N-1} \tau\left\|f_{h}^{n+1}\right\|_{L}^{2}+\left\|\pi_{h} \widetilde{u}_{h}^{0}\right\|_{L}^{2}+\tau^{2}\left\|\nabla p_{h}^{0}\right\|_{L}^{2},
\end{aligned}
$$

provided the standard hyperbolic CFL condition holds with

$$
\text { Co }<\frac{1}{4}\left(C_{4}^{2} \gamma_{1} \check{c}(\beta, h)\right)^{-1},
$$

for the explicit treatment of the velocity, and the following additional condition holds:

$$
C_{2}^{2} \gamma_{4} \check{c}(\beta, h) h \leq \tau,
$$

for the explicit treatment of the pressure with $C_{2}$ defined by (24).

$\mathrm{RR} \mathrm{n}^{\circ} 8799$ 
Proof. In the implicit case where $u^{*}=u_{h}^{n+1}$ and $p^{*}=p_{h}^{n+1}$, there is only one term in the right-hand side of $(27)$ since the stabilization terms vanish. We obtain that

$$
\begin{aligned}
\sum_{n=0}^{N-1} \tau\left(f_{h}^{n+1}, u_{h}^{n+1}\right)_{L} & =\sum_{n=0}^{N-1} \tau\left(f_{h}^{n+1}, \widetilde{u}_{h}^{n+1}\right)_{L}+\tau^{2} \sum_{n=0}^{N-1}\left(f_{h}^{n+1}, \nabla\left(p_{h}^{n+1}-p_{h}^{n}\right)\right)_{L} \\
& \lesssim \sum_{n=0}^{N-1} \tau\left\|f_{h}^{n+1}\right\|_{L}^{2}+\sum_{n=0}^{N-1} \tau\left\|\pi_{h} \widetilde{u}_{h}^{n+1}\right\|_{L}^{2}+\sum_{n=0}^{N-1} \tau^{3}\left\|\nabla p_{h}^{n+1}\right\|_{L}^{2}
\end{aligned}
$$

and (36) is an immediate consequence of the discrete Gronwall's lemma, assuming $\tau$ sufficiently small so that the term $\left\|\pi_{h} \widetilde{u}_{h}^{N}\right\|^{2}+\tau^{2}\left\|\nabla p_{h}^{N}\right\|_{L}^{2}$ in the righ-hand side can be absorbed in the lefthand side. Taking $u_{h}^{*}=\pi_{h} \widetilde{u}_{h}^{n}$, the second term on the right-hand side of (27) is absorbed by the third term in the left-hand side owing to 37 since

$$
\tau\left(S_{h}^{u}\left(u_{h}^{n+1}-\pi_{h} \widetilde{u}_{h}^{n}\right), u_{h}^{n+1}\right) \leq C_{4}^{2} \gamma_{1} \check{c}(\beta, h) \operatorname{Co}\left\|u_{h}^{n+1}-\pi_{h} \widetilde{u}_{h}^{n}\right\|_{L}^{2}+\frac{1}{4} \tau\left|u_{h}^{n+1}\right|_{S^{u}}^{2}
$$

Taking $u_{h}^{*}=u_{h}^{n}$ is feasible for piecewise linears. To see this recall that by equation (29), $u_{h}^{n}=$ $\pi_{h} \widetilde{u}_{h}^{n}-\tau \pi_{h} \nabla\left(p_{h}^{n+1}-p_{h}^{n}\right)$. For $k=1$, the following holds:

$$
\begin{aligned}
\left(S_{h}^{u}\left(u_{h}^{n+1}-u_{h}^{n}\right), u_{h}^{n+1}\right)_{L}=\left(S_{h}^{u}\left(u_{h}^{n+1}-\pi_{h} \widetilde{u}_{h}^{n}\right), u_{h}^{n+1}\right)_{L} & \\
& +\left(S_{h}^{u} \tau\left(I-\pi_{h}\right) \nabla\left(p_{h}^{n+1}-p_{h}^{n}\right), u_{h}^{n+1}\right),
\end{aligned}
$$

since the elementwise gradient of $\nabla\left(p_{h}^{n+1}-p_{h}^{n}\right)$ vanishes. The first contribution on the right-hand side is absorbed by the third term in the left-hand side of (27) using (37) as before. For the second term, we deduce from (35) that

$$
\begin{aligned}
\tau\left(S_{h}^{u} \tau\left(I-\pi_{h}\right) \nabla\left(p_{h}^{n+1}-p_{h}^{n}\right), u_{h}^{n+1}\right) \leq \tau \frac{1}{4}\left|u_{h}^{n+1}\right|_{S^{u}}^{2} & \\
& \quad+C_{4}^{2} \gamma_{1} \check{c}(\beta, h) \operatorname{Co}\left\|\tau\left(I-\pi_{h}\right) \nabla\left(p_{h}^{n+1}-p_{h}^{n}\right)\right\|_{L}^{2} .
\end{aligned}
$$

The first term can be absorbed by the contribution from the velocity stabilization in the left-hand side, and the second one can be absorbed by the second to last term in the left-hand side of (27) still using 37. Finlly, taking $p_{h}^{*}=p_{h}^{n}$, we observe that

$$
\begin{aligned}
\tau\left(S_{h}^{p}\left(p_{h}^{n+1}-p_{h}^{n}\right), p_{h}^{n+1}\right)_{L} & =\tau\left(\left(I-\pi_{h}\right) \nabla\left(p_{h}^{n+1}-p_{h}^{n}\right), \widetilde{S}_{h}^{p} p_{h}^{n+1}\right)_{L} \\
& \leq \tau^{2}\left\|\left(I-\pi_{h}\right) \nabla\left(p_{h}^{n+1}-p_{h}^{n}\right)\right\|^{2}+C_{2}^{2} \gamma_{4} \check{c}(\beta, h) h\left|p_{h}^{n+1}\right|_{S^{p}}^{2},
\end{aligned}
$$

and the last term can be absorbed in the left-hand side using (38).

Remark 4.1 (Reverse CFL). For high-Reynolds flows, condition (37) yields a reverse hyperbolic CFL condition; it can be made compatible with other bounds on the Courant number by choosing $\gamma_{4}$ small enough. In the low-Reynolds regime, this condition becomes milder, of the form $h^{2} \lesssim \mu \tau$.

Remark 4.2. Since we use equal-order interpolation, the projection step, which is equivalent to a Darcy problem, has inf-sup constant that tends to zero as $h \rightarrow 0$ unless stabilization is applied. Corllary 4.1 shows that applying the stabilization operator with the pressure from the previous time step is enough to restore uniform inf-sup stability. 
Remark 4.3. It also follows from (36) that control of $\pi_{h} \widetilde{u}_{h}^{N}$ is sufficient to control $u_{h}^{N}$, which justifies the analysis in the variable $\pi_{h} \widetilde{u}_{h}^{N}$. Indeed, let us first note that since (36) holds for all $N$, we infer that

$$
\max _{n \in\{0, \ldots, N\}}\left\|\pi_{h} \widetilde{u}_{h}^{n}\right\|_{L}^{2}+\max _{n \in\{0, \ldots, N\}} \tau^{2}\left\|\nabla p_{h}^{n}\right\|_{L}^{2} \lesssim \sum_{n=0}^{N-1} \tau\left\|f_{h}^{n+1}\right\|_{L}^{2}+\frac{1}{2}\left\|\pi_{h} \widetilde{u}_{h}^{0}\right\|_{L}^{2}+\frac{1}{2} \tau^{2}\left\|\nabla p_{h}^{0}\right\|_{L}^{2} .
$$

Owing to 29 we infer that

$$
\begin{aligned}
\left\|u_{h}^{N}\right\|_{L}^{2} \lesssim & \left\|\pi_{h} \widetilde{u}_{h}^{N}\right\|_{L}^{2}+\tau^{2}\left\|\nabla p_{h}^{N}\right\|_{L}^{2}+\tau^{2}\left\|\nabla p_{h}^{N-1}\right\|_{L}^{2} \\
& \lesssim \max _{n \in\{0, \ldots, N\}}\left\|\pi_{h} \widetilde{u}_{h}^{n}\right\|_{L}^{2}+\max _{n \in\{0, \ldots, N\}} \tau^{2}\left\|\nabla p_{h}^{n}\right\|_{L}^{2}
\end{aligned}
$$

which leads to the desired stability bound on $\left\|u_{h}^{N}\right\|_{L}^{2}$ and later to the same error estimates for $\left\|u_{h}^{N}\right\|_{L}^{2}$ as for $\left\|\pi_{h} \widetilde{u}_{h}^{N}\right\|_{L}^{2}$. Hence, it suffices to consider the variable $\pi_{h} \widetilde{u}_{h}^{N}$, keeping in mind that all the estimates carry over without modification to $u_{h}^{N}$.

\subsection{Error analysis}

The error analysis follows in a relatively straightforward fashion, using the stability result (36), followed by consistency (Galerkin orthogonality) and the boundedness result from Lemma 2.1 . The order in time is a consequence of the truncation error of the BDF1 scheme and the first-order splitting error of the incremental pressure-projection method. We first introduce a suitable error equation, with approximation errors and truncation errors as data. Then we apply the stability result from the previous section to this error equation, repeating some steps for enhanced clarity. The error estimates are then obtained using consistency followed by boundedness; convergence rates finally result from the approximation properties of finite elements.

To derive the error equation, we set

$$
\begin{array}{ll}
e_{h}^{n}:=u_{h}^{n}-\pi_{h} u^{n}, & e_{\pi}^{n}:=u^{n}-\pi_{h} u^{n}, \\
\eta_{h}^{n}:=p_{h}^{n}-\pi_{h} p^{n}, & \eta_{\pi}^{n}:=p^{n}-\pi_{h} p^{n},
\end{array}
$$

together with $\widetilde{e}_{h}^{n}:=\widetilde{u}_{h}^{n}-\pi_{h} u^{n}$ and $\widetilde{e}_{\pi}^{n}:=e_{\pi}^{n}$. We introduce the truncation errors

$$
\Psi_{1}^{n}:=\partial_{t} u^{n}-\frac{1}{\tau}\left(u^{n+1}-u^{n}\right), \quad \Psi_{2}^{n}:=p^{n+1}-p^{n} .
$$

Finally, we set

$$
\zeta_{h}^{n}:=\eta_{h}^{n}-\pi_{h} \Psi_{2}^{n},
$$

so that $\zeta_{h}^{n}=p_{h}^{n}-\pi_{h} p^{n+1}$. Owing to Remark 4.3 we infer that

$$
\left\|\widetilde{e}_{h}^{n}\right\|_{L} \leq\left\|e_{h}^{n}\right\|_{L}+\tau\left\|\nabla \eta_{h}^{n}\right\|_{L}+\tau\left\|\nabla \eta_{h}^{n-1}\right\|_{L}+\tau\left\|\nabla \pi_{h} \Psi_{2}^{n-1}\right\|_{L} .
$$

We also recall the following standard truncation error estimates

$$
\sum_{n=1}^{N} \tau\left(\left\|\Psi_{1}^{n}\right\|_{L}^{2}+\left\|\nabla \Psi_{2}^{n}\right\|_{L}^{2}\right) \lesssim \tau^{2}\left(\left\|\partial_{t}^{2} u\right\|_{\ell^{2}\left(0, t_{\mathrm{F}} ; L\right)}^{2}+\left\|\partial_{t} \nabla p\right\|_{\ell^{2}\left(0, t_{\mathrm{F}} ; L\right)}^{2}\right)
$$

$\mathrm{RR} \mathrm{n}^{\circ} 8799$ 
Lemma 4.2 (Error equations). The velocity error equation takes the form

$$
\frac{1}{\tau}\left(e_{h}^{n+1}-\pi_{h} \widetilde{e}_{h}^{n}\right)+A_{h} e_{h}^{n+1}+S_{h}^{u}\left(e_{h}^{*}\right)+B_{h}^{\mathrm{T}} \zeta_{h}^{n}=B_{h}^{\mathrm{T}} \eta_{\pi}^{n+1}+\pi_{h} \Psi_{1}^{n}+A_{h} e_{\pi}^{n+1}+S_{h}^{u} e_{\pi}^{*},
$$

with $e_{h}^{*}=e_{h}^{n+1}$ and $e_{\pi}^{*}=e_{\pi}^{n+1}$ for implicit velocity stabilization, while $e_{h}^{*}=\pi_{h} \widetilde{e}_{h}^{n}$ or $e_{h}^{*}=e_{h}^{n}$ and $e_{\pi}^{*}=e_{\pi}^{n}$ for explicit velocity stabilization. Moreover, the pressure error equation takes the form

$$
\begin{aligned}
\frac{1}{\tau}\left(\widetilde{e}_{h}^{n+1}-e_{h}^{n+1}\right)+C_{h}^{\mathrm{T}}\left(\eta_{h}^{n+1}-\zeta_{h}^{n}\right)+\frac{1}{\tau} \widetilde{S}_{h}^{p} \eta_{h}^{*} & =\frac{1}{\tau} \widetilde{S}_{h}^{p} \eta_{\pi}^{*}, \\
C_{h} \widetilde{e}_{h}^{n+1} & =C_{h} e_{\pi}^{n+1},
\end{aligned}
$$

where $\eta_{h}^{*}=\eta_{h}^{n+1}$ and $\eta_{\pi}^{*}=\eta_{\pi}^{n+1}$ for implicit pressure stabilization and $\eta_{h}^{*}=\eta_{h}^{n}$ and $\eta_{\pi}^{*}=\eta_{\pi}^{n}$ for explicit pressure stabilization.

Proof. Use the fact that $\pi_{h} \partial_{t} u^{n+1}+A_{h} u^{n+1}+B_{h}^{\mathrm{T}} p^{n+1}=f_{h}^{n+1}$ to prove 45. Moreover, 46a is directly verified by adding and subtracting $\pi_{h} u^{n+1}$ and $\pi_{h} p^{n+1}$. Finally, use the fact that $C_{h} u^{n+1}=0$ to prove $46 \mathrm{~b}$.

Theorem 4.1 (Error estimate). Assume that $(u, p) \in\left[H^{k+1}(\Omega)\right]^{d+1}$ for the solution of (1). Let $u_{h}^{n}$ and $p_{h}^{n}$ be the solutions of $(19)-(20 \mathrm{~b}), n=1, \ldots, N$, with mesh-parameters satisfying the assumptions of Corollary 4.1 if an explicit treatment of stabilization is employed. Then the following holds with $e^{n}:=u_{h}^{n}-u\left(t^{n}\right), \eta^{n}:=p_{h}^{n}-p\left(t^{n}\right)$ and $\bar{\eta}^{N}:=N^{-1} \sum_{n=1}^{N} \eta^{n}$ :

$$
\begin{aligned}
& \left\|e^{N}\right\|_{L}+\tau\left\|\nabla \bar{\eta}^{N}\right\|_{L}+\left(\sum_{n=1}^{N} \tau\left(\left\|e^{n}\right\|_{\mu, \beta}^{2}+\left|e^{n}\right|_{S^{u}}^{2}+|\eta|_{S^{p}}^{2}\right)\right)^{\frac{1}{2}} \\
& \lesssim\left(c(\beta, h)^{\frac{1}{2}}+\check{c}(\beta, h)^{\frac{1}{2}} \beta_{\Omega}+t_{\mathrm{F}}^{\frac{1}{2}} L_{\beta} h^{\frac{1}{2}}\right) h^{k+\frac{1}{2}}\|u\|_{\ell^{2}\left(0, t_{F} ; H^{k+1}(\Omega)\right)} \\
& +\check{c}(\beta, h)^{\frac{1}{2}} h^{k+\frac{1}{2}}\|p\|_{\ell^{2}\left(0, t_{F} ; H^{k+1}(\Omega)\right)}+t_{F}^{\frac{1}{2}} \tau\left(\left\|\partial_{t}^{2} u\right\|_{L, Q}+\left\|\partial_{t} \nabla p\right\|_{L, Q}\right), \\
& \left\|\bar{\eta}^{N}\right\|_{L} \lesssim h^{k+1}\left\|\bar{p}^{N}\right\|_{H^{k+1}(\Omega)} \\
& +t_{\mathrm{F}}^{-\frac{1}{2}}\left(c(\beta, h)^{\frac{1}{2}}+\check{c}(\beta, h)^{\frac{1}{2}} \beta_{\Omega}+t_{\mathrm{F}}^{\frac{1}{2}} L_{\beta} h^{\frac{1}{2}}\right) h^{k+\frac{1}{2}}\|u\|_{\ell^{2}\left(0, t_{F} ; H^{k+1}(\Omega)\right)} \\
& +t_{\mathrm{F}}^{-\frac{1}{2}} \check{c}(\beta, h)^{\frac{1}{2}} h^{k+\frac{1}{2}}\|p\|_{\ell^{2}\left(0, t_{F} ; H^{k+1}(\Omega)\right)}+\tau\left(\left\|\partial_{t}^{2} u\right\|_{L, Q}+\left\|\partial_{t} \nabla p\right\|_{L, Q}\right) \text {. }
\end{aligned}
$$

Proof. After a standard decomposition of the error in an approximation part $e_{\pi}^{n}, \eta_{\pi}^{n}$ and a discrete part $e_{h}^{n}, \eta_{h}^{n}$ and recalling equation (43), we observe that it is enough to bound for all $N$ the discrete error

$$
\left\|\pi_{h} \widetilde{e}_{h}^{N}\right\|_{L}^{2}+\tau^{2}\left\|\nabla \eta_{h}^{N}\right\|_{L}^{2}+\tau \sum_{n=1}^{N}\left(\left\|e_{h}^{n+1}\right\|_{\mu, \beta}^{2}+\left|e_{h}^{n+1}\right|_{S^{u}}^{2}+\tau\left|\eta_{h}^{n+1}\right|_{S^{p}}^{2}\right) .
$$

Step 1. Multiplying 45 by $\tau e_{h}^{n+1}$ yields

$$
\begin{aligned}
\frac{1}{2}\left\|e_{h}^{n+1}\right\|_{L}^{2}-\frac{1}{2}\left\|\pi_{h} \widetilde{e}_{h}^{n}\right\|_{L}^{2}+\frac{1}{2}\left\|e_{h}^{n+1}-\pi_{h} \widetilde{e}_{h}^{n}\right\|_{L}^{2}+\tau\left(A_{h} e_{h}^{n+1}, e_{h}^{n+1}\right)_{L}^{2}+\tau\left|e_{h}^{n+1}\right|_{S^{u}}^{2}= \\
-\tau\left(B_{h}^{\mathrm{T}} \zeta_{h}^{n}, e_{h}^{n+1}\right)_{L}+\mathfrak{T}_{1}+\ldots+\mathfrak{T}_{5},
\end{aligned}
$$


with

$$
\begin{aligned}
& \mathfrak{T}_{1}=\tau\left(B_{h}^{\mathrm{T}} \eta_{\pi}^{n+1}, e_{h}^{n+1}\right)_{L}, \\
& \mathfrak{T}_{2}=\tau\left(\Psi_{1}^{n}, e_{h}^{n+1}\right)_{L}, \\
& \mathfrak{T}_{3}=\tau\left(S_{h}^{u}\left(e_{h}^{n+1}-e_{h}^{*}\right), e_{h}^{n+1}\right)_{L}, \\
& \mathfrak{T}_{4}=\tau\left(A_{h} e_{\pi}^{n+1}, e_{h}^{n+1}\right)_{L}, \\
& \mathfrak{T}_{5}=\tau\left(S_{h}^{u} e_{\pi}^{*}, e_{h}^{n+1}\right)_{L} .
\end{aligned}
$$

$\mathfrak{T}_{1}$ is handled using equation $(13 \mathrm{~b}$, exploiting the stabilization on the divergence and on the normal component at the boundary, followed by Young's inequality, absorbing the term $\tau\left|e_{h}^{n+1}\right|_{S^{u}}^{2}$ in the left-hand side. $\mathfrak{T}_{2}$ is handled by Gronwall's inequality; $\mathfrak{T}_{3}$ is handled as in Section 4.2 . depending on the choice of velocity stabilization; $\mathfrak{T}_{4}$ is controlled using $(13 \mathrm{c})$, leading to the term $\tau\left(\left\|e_{h}^{n+1}\right\|_{\mu, \beta}^{2}+\left|e_{h}^{n+1}\right|_{S^{u}}^{2}\right)$ that we absorb in the left-hand side and a term of the form $L_{\beta}\left\|e_{h}^{n+1}\right\|_{L}^{2}$ that is treated using Gronwall's inequality. Finally $\mathfrak{T}_{5}$ is controlled using a Cauchy-Schwarz inequality and Young's inequality and absorbing the term $\tau\left|e_{h}^{n+1}\right|_{S^{u}}^{2}$ in the left-hand side.

Step 2. Applying $\pi_{h}$ to $46 \mathrm{a}$ yields

$$
\left(\pi_{h} \widetilde{e}_{h}^{n+1}-e_{h}^{n+1}\right)+\tau B_{h}^{\mathrm{T}}\left(\eta_{h}^{n+1}-\zeta_{h}^{n}\right)=0 .
$$

Applying $B_{h}$ to this equation leads to

$$
B_{h} \pi_{h} \widetilde{e}_{h}^{n+1}-B_{h} e_{h}^{n+1}+\tau B_{h} B_{h}^{\mathrm{T}}\left(\eta_{h}^{n+1}-\zeta_{h}^{n}\right)=0 .
$$

Applying $C_{h}$ to $46 \mathrm{a}$ and using $46 \mathrm{~b}$, we infer that

$$
C_{h} e_{\pi}^{n+1}-B_{h} e_{h}^{n+1}+\tau C_{h} C_{h}^{\mathrm{T}}\left(\eta_{h}^{n+1}-\zeta_{h}^{n}\right)+S_{h}^{p}\left(\eta_{h}^{*}-\eta_{\pi}^{*}\right)=0 .
$$

Subtracting (52) and (51),

$$
B_{h} \pi_{h} \widetilde{e}_{h}^{n+1}=\tau\left(C_{h} C_{h}^{\mathrm{T}}-B_{h} B_{h}^{\mathrm{T}}\right)\left(\eta_{h}^{n+1}-\zeta_{h}^{n}\right)+C_{h} e_{\pi}^{n+1}+S_{h}^{p}\left(\eta_{h}^{*}-\eta_{\pi}^{*}\right) .
$$

Testing with $\zeta_{h}^{n}$ yields

$$
\begin{aligned}
\frac{1}{2} \tau^{2}\left\|\nabla \eta_{h}^{n+1}\right\|_{L}^{2}-\frac{1}{2} \tau^{2}\left\|\nabla \zeta_{h}^{n}\right\|_{L}^{2}-\frac{1}{2} \tau^{2}\left\|\nabla\left(\eta_{h}^{n+1}-\zeta_{h}^{n}\right)\right\|_{L}^{2} & +\tau\left(S_{h}^{p}\left(\eta_{h}^{*}-\eta_{\pi}^{*}\right), \zeta_{h}^{n}\right)_{L} \\
& =\tau\left(B_{h}^{\mathrm{T}} \zeta_{h}^{n}, e_{h}^{n+1}\right)_{L}-\tau\left(C_{h} e_{\pi}^{n+1}, \zeta_{h}^{n}\right)_{L}
\end{aligned}
$$

Step 3. Testing 50 with $\pi_{h} \widetilde{e}_{h}^{n+1}$ yields

$$
\frac{1}{2}\left\|\pi_{h} \widetilde{e}_{h}^{n+1}\right\|_{L}^{2}-\frac{1}{2}\left\|e_{h}^{n+1}\right\|_{L}^{2}+\frac{1}{2}\left\|\pi_{h} \widetilde{e}_{h}^{n+1}-e_{h}^{n+1}\right\|_{L}^{2}=-\tau\left(B_{h}^{\mathrm{T}}\left(\eta_{h}^{n+1}-\zeta_{h}^{n}\right), \pi_{h} \widetilde{e}_{h}^{n+1}\right)_{L},
$$

and using

$$
\left\|\pi_{h} \widetilde{e}_{h}^{n+1}-e_{h}^{n+1}\right\|_{L}^{2}=\tau^{2}\left\|\pi_{h} \nabla\left(\eta_{h}^{n+1}-\zeta_{h}^{n}\right)\right\|_{L}^{2}
$$

together with 53 for the right-hand side, we arrive at

$$
\begin{array}{r}
\frac{1}{2}\left\|\pi_{h} \widetilde{e}_{h}^{n+1}\right\|_{L}^{2}-\frac{1}{2}\left\|e_{h}^{n+1}\right\|_{L}^{2}+\frac{1}{2} \tau^{2}\left\|\pi_{h} \nabla\left(\eta_{h}^{n+1}-\zeta_{h}^{n}\right)\right\|_{L}^{2}=-\tau^{2}\left\|\left(I-\pi_{h}\right) \nabla\left(\eta_{h}^{n+1}-\zeta_{h}^{n}\right)\right\|_{L}^{2} \\
-\tau\left(C_{h} e_{\pi}^{n+1}, \eta_{h}^{n+1}-\zeta_{h}^{n}\right)_{L}-\tau\left(S_{h}^{p}\left(\eta_{h}^{*}-\eta_{\pi}^{*}\right), \eta_{h}^{n+1}-\zeta_{h}^{n}\right)_{L} .
\end{array}
$$

$\mathrm{RR} \mathrm{n}^{\circ} 8799$ 
Step 4. Final combination. We infer that

$$
\begin{aligned}
& \frac{1}{2}\left\|\pi_{h} \widetilde{e}_{h}^{n+1}\right\|_{L}^{2}-\frac{1}{2}\left\|\pi_{h} \widetilde{e}_{h}^{n}\right\|_{L}^{2}+\frac{1}{2}\left\|e_{h}^{n+1}-\pi_{h} \widetilde{e}_{h}^{n}\right\|_{L}^{2}+\tau\left\|e_{h}^{n+1}\right\|_{A}^{2}+\tau\left|e_{h}^{n+1}\right|_{S^{u}}^{2} \\
& +\frac{1}{2} \tau^{2}\left\|\nabla \eta_{h}^{n+1}\right\|_{L}^{2}-\frac{1}{2} \tau^{2}\left\|\nabla \zeta_{h}^{n}\right\|_{L}^{2}+\frac{1}{2} \tau^{2}\left\|\left(I-\pi_{h}\right) \nabla\left(\eta_{h}^{n+1}-\zeta_{h}^{n}\right)\right\|_{L}^{2}+\tau\left|\eta_{h}^{n+1}\right|_{S^{p}}^{2} \\
& =\mathfrak{T}_{1}+\ldots+\mathfrak{T}_{8},
\end{aligned}
$$

with

$$
\begin{aligned}
& \mathfrak{T}_{6}=-\tau\left(C_{h} e_{\pi}^{n+1}, \eta_{h}^{n+1}\right)_{L}, \\
& \mathfrak{T}_{7}=\tau\left(S_{h}^{p} \eta_{h}^{n+1}, \eta_{\pi}^{*}\right)_{L}, \\
& \mathfrak{T}_{8}=\tau\left(S_{h}^{p} \eta_{h}^{n+1}, \eta_{h}^{n+1}-\eta_{h}^{*}\right)_{L} .
\end{aligned}
$$

The lack of a telescoping form for $\frac{1}{2} \tau^{2}\left\|\nabla \eta_{h}^{n+1}\right\|_{L}^{2}-\frac{1}{2} \tau^{2}\left\|\nabla \zeta_{h}^{n}\right\|_{L}^{2}$ is not a problem since by a triangle inequality, we infer that

$$
\left\|\nabla \zeta_{h}^{n}\right\|_{L}^{2} \leq(1+\tau)\left\|\nabla \eta_{h}^{n}\right\|_{L}^{2}+\left(1+\tau^{-1}\right)\left\|\nabla \pi_{h} \Psi_{2}^{n}\right\|_{L}^{2}
$$

the first term is handled by Gronwall's inequality and the second yields an $O(\tau)$-error. The term $\mathfrak{T}_{6}$ is bounded using (13a) and the term $\mathfrak{T}_{7}$ by the Cauchy-Schwarz inequality. The pressure stabilization contributions are then absorbed in the left-hand side. The term $\mathfrak{T}_{8}$ is treated as detailed in Section 4.2 ,

Collecting the above bounds, we obtain the inequality

$$
\begin{aligned}
\left\|\pi_{h} \widetilde{e}_{h}^{n+1}\right\|_{L}^{2}- & \left\|\pi_{h} \widetilde{e}_{h}^{n}\right\|_{L}^{2}+\tau^{2}\left\|\nabla \eta_{h}^{n+1}\right\|_{L}^{2}-\tau^{2}\left\|\nabla \eta_{h}^{n}\right\|_{L}^{2} \\
& +\tau\left\|e_{h}^{n+1}\right\|_{\mu, \beta}^{2}+\tau\left|e_{h}^{n+1}\right|_{S^{u}}^{2}+\tau\left|\eta_{h}^{n+1}\right|_{S^{p}}^{2} \\
\lesssim & \tau \check{c}(\beta, h)\left(h^{-1}\left\|\eta_{\pi}^{n+1}\right\|_{L}^{2}+\left\|\eta_{\pi}^{n+1}\right\|_{L, \partial \Omega}^{2}\right) \\
& +\tau\left(c\left(\beta, h_{F}\right) h^{-1}+L_{\beta}^{2} t_{F}\right)\left\|e_{\pi}^{n+1}\right\|_{L}^{2} \\
& +\tau\left\|e_{\pi}^{n+1}\right\|_{\mu, \beta}^{2}+\tau\left(\left|e_{\pi}^{*}\right|_{S^{u}}^{2}+\left|\eta_{\pi}^{*}\right|_{S^{p}}^{2}\right)+\tau t_{F}\left(\left\|\Psi_{1}^{n}\right\|_{L}^{2}+\left\|\nabla \Psi_{2}^{n}\right\|_{L}^{2}\right) \\
& +\tau t_{F}^{-1}\left(\left\|e_{h}^{n+1}\right\|_{L}^{2}+\tau^{2}\left\|\nabla \eta_{h}^{n+1}\right\|_{L}^{2}\right) .
\end{aligned}
$$

We add and subtract $\pi_{h} \widetilde{e}_{h}^{n+1}$ in $\left\|e_{h}^{n+1}\right\|_{L}^{2}$ in the last term in the right-hand side, and we use a triangle inequality, (54), and (55). We obtain inequality

$$
\begin{aligned}
& \tau t_{F}^{-1}\left(\left\|e_{h}^{n+1}\right\|_{L}^{2}+\tau^{2}\left\|\nabla \eta_{h}^{n+1}\right\|_{L}^{2}\right) \\
& \quad \leq 2 \tau t_{F}^{-1}\left(\left\|\pi_{h} \widetilde{e}_{h}^{n+1}\right\|_{L}^{2}+\left\|\pi_{h} \widetilde{e}_{h}^{n+1}-e_{h}^{n+1}\right\|_{L}^{2}+\tau^{2}\left\|\nabla \eta_{h}^{n+1}\right\|_{L}^{2}\right) \\
& \quad \leq 2 \tau t_{F}^{-1}\left(\left\|\pi_{h} \widetilde{e}_{h}^{n+1}\right\|_{L}^{2}+5(1+\tau) \tau^{2}\left\|\nabla \eta_{h}^{n+1}\right\|_{L}^{2}+2\left(1+\tau^{-1}\right) \tau^{2}\left\|\nabla \pi_{h} \Psi_{2}^{n}\right\|_{L}^{2}\right) .
\end{aligned}
$$

Now assume that $\tau$ is sufficiently small so that the $\left\|\pi_{h} \widetilde{e}_{h}^{N}\right\|_{L}^{2}+\tau^{2}\left\|\nabla \eta_{h}^{N}\right\|_{L}^{2}$ contribution of the right hand side can be absorbed in the left hand side. Then the velocity error estimate follows after summing over $n$, applying Gronwall's Lemma and bounding the approximation errors using Lemma 2.2 .

To prove the estimate on the time-averaged $L^{2}$-error on the pressure, we observe that the following error representation holds for all $1 \leq n \leq N-1$ :

$$
\begin{array}{r}
\pi_{h} e^{n+1}-\pi_{h} e^{n}+\tau A_{h} e^{n+1}+\tau S_{h}^{u}\left(u_{h}^{*}\right)=\pi_{h}\left(u\left(t^{n+1}\right)-u\left(t^{n}\right)-\tau \partial_{t} u\left(t^{n+1}\right)\right)-\tau B_{h}^{\mathrm{T}} \eta^{n+1} \\
+\tau B_{h}^{\mathrm{T}}\left(\eta^{n+1}-2 \eta^{n}+\eta^{n-1}\right)+\tau B_{h}^{\mathrm{T}}\left(p\left(t^{n+1}\right)-2 p\left(t^{n}\right)+p\left(t^{n-1}\right)\right) .
\end{array}
$$


Following the arguments of Theorem 3.1, we let $\bar{v}_{p} \in\left[H_{0}^{1}(\Omega)\right]^{d}$ be such that

$$
\nabla \cdot \bar{v}_{p}=\bar{\eta}^{N}, \quad\left\|\bar{v}_{p}\right\|_{H^{1}(\Omega)} \lesssim\left\|\bar{\eta}^{n}\right\|_{L} .
$$

Then using $y_{h}^{n}=\pi_{h} p^{n}-p_{h}^{n}$ we infer that

$$
\begin{aligned}
& \left\|\bar{\eta}^{N}\right\|_{L}^{2}=\left(\nabla\left(\bar{p}^{n}-\bar{\pi}_{h} p^{n}\right), \bar{v}_{p}-\pi_{h} \bar{v}_{p}\right)_{L}+\left(\bar{y}_{h}^{n}, C_{h}\left(\bar{v}_{p}-\pi_{h} \bar{v}_{p}\right)\right)_{L} \\
& +t_{F}^{-1} \tau \sum_{n=1}^{N-1}\left[\left(\partial_{\tau}^{1} e^{n+1}+A_{h} e^{n+1}+S_{h}^{u} u_{h}^{*}, \pi_{h} \bar{v}_{p}\right)_{L}+\left(\partial_{t} u\left(t^{n}\right)-\tau^{-1}\left(u\left(t^{n}\right)-u\left(t^{n-1}\right), \pi_{h} \bar{v}_{p}\right)_{L}\right.\right. \\
& \quad-\left(B_{h}^{\mathrm{T}}\left(\eta^{n+1}-2 \eta^{n}+\eta^{n-1}\right), \pi_{h} \bar{v}_{p}\right)_{L}+\left(B_{h}^{\mathrm{T}}\left(p\left(t^{n+1}\right)-2 p\left(t^{n}\right)+p\left(t^{n-1}\right), \pi_{h} \bar{v}_{p}\right)_{L}\right] .
\end{aligned}
$$

The only terms that differ from the monolithic case are those in the last line. We observe that telescoping the sum and using Poincaré's inequality leads to

$$
\begin{gathered}
\tau \sum_{n=1}^{N-1}\left(B_{h}^{\mathrm{T}}\left(\eta^{n+1}-2 \eta^{n}+\eta^{n-1}\right), \pi_{h} \bar{v}_{p}\right)_{L}=\tau\left(B_{h}^{\mathrm{T}}\left(\eta^{N}+\eta^{N-1}-\eta^{1}+\eta^{0}\right), \pi_{h} \bar{v}_{p}\right)_{L} \\
\lesssim \tau\left(\left\|\nabla \eta^{N}\right\|_{L}+\left\|\nabla \eta^{N-1}\right\|_{L}\right)\left\|\pi_{h} \bar{v}_{p}\right\|_{H^{1}(\Omega)} \\
+\tau\left(\pi_{h} p\left(t^{1}\right)-p\left(t^{1}\right)-\pi_{h} p\left(t^{0}\right)+p\left(t^{0}\right), \nabla \cdot \pi_{h} \bar{v}_{p}\right)_{L} \\
-\tau\left(\pi_{h} p\left(t^{1}\right)-p\left(t^{1}\right)-\pi_{h} p\left(t^{0}\right)+p\left(t^{0}\right), \nu \cdot\left(\pi_{h} \bar{v}_{p}-\bar{v}_{p}\right)\right)_{L, \partial \Omega} \\
\lesssim \tau\left(\left\|\nabla \eta^{N}\right\|_{L}+\left\|\nabla \eta^{N-1}\right\|_{L}+h\left\|\nabla p\left(t^{1}\right)\right\|_{L}+h\left\|\nabla p_{0}\right\|_{L}\right)\left\|\bar{v}_{p}\right\|_{H^{1}(\Omega)},
\end{gathered}
$$

where $\tau\left(\left\|\nabla \eta^{N}\right\|_{L}+\left\|\nabla \eta^{N-1}\right\|_{L}\right)$ is bounded in the first part of the proof. We use that $\left\|\nabla p\left(t^{1}\right)\right\|_{L}+$ $\left\|\nabla p_{0}\right\|_{L} \lesssim\left\|\partial_{t} \nabla p\right\|_{L, Q}$ to conclude.

\section{$5 \quad$ Numerical results}

We illustrate the theoretical results on three test cases at different Reynolds numbers. In all cases we consider the Navier-Stokes equations in two space dimensions with unit density. The convective term is handled using extrapolation, taking the form $\left(u^{*} \cdot \nabla\right) u_{h}^{n+1}$ with $u^{*}=u_{h}^{n}$ or $u^{*}=2 u_{h}^{n}-u_{h}^{n-1}$. No fixed point iterations are performed. We have considered the following test cases:

- academic $2 \mathrm{D}$ solution at $\operatorname{Re}=10^{5}$;

- flow around a cylinder at $\operatorname{Re}=10^{2}$;

- Kelvin-Helmholtz instability at $\mathrm{Re}=10^{4}$.

Numerical tests not reported here indicate that the tuning of the discretization parameters when the pressure stabilization is treated explicitly so that the method is both stable and accurate in all regimes are too delicate to be of practical use. Therefore, we do not report results with an explicit treatment of the pressure stabilization. Instead, an example showing the effect of explicit treatment of the velocity stabilization on the accuracy is presented. Although the theoretical results for the fractional-step method presented above are valid only for first-order BDF1 time scheme, we present numerical examples also for the BDF2 time scheme. Observe that the stability result from Lemma 4.1 remains valid in this case, but the order of the scheme is not improved due to the first-order splitting error.

$\mathrm{RR} \mathrm{n}^{\circ} 8799$ 


\subsection{Academic 2D solution at $\mathrm{Re}=10^{5}$}

This academic test case was used in [13] to show the effect of stabilization on the accuracy of a projection-based fractional-step solver for the Navier-Stokes equations. Let $\Omega$ be the unit square and set

$$
\left\{\begin{aligned}
u_{\mathrm{ex}} & =u(x, y) g(t), \quad p_{\mathrm{ex}}=-\frac{1}{4}(\cos (2 x)+\cos (2 y)) g^{2}(t), \\
u(x, y) & =(-\cos (x) \sin (y), \sin (x) \cos (y))^{\mathrm{T}}, \quad g(t)=\sin (2 t) .
\end{aligned}\right.
$$

It is straightforward to verify that this is the exact solution of the Navier-Stokes equations

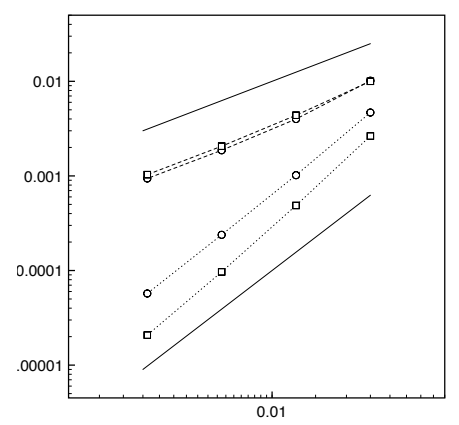

(a)

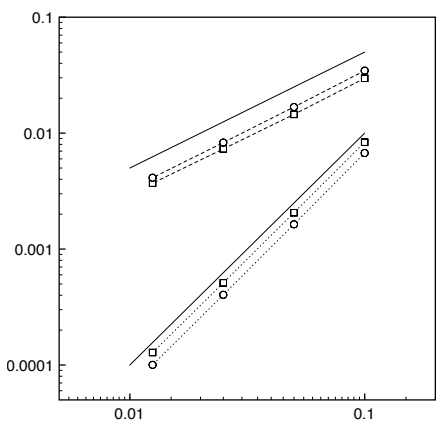

(b)

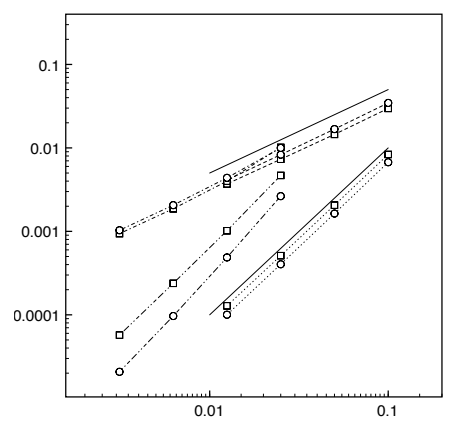

(c)

Figure 1: Convergence plot for the fractional-step method applied to the problem defined by the exact solution (57). (a) explicit velocity stabilization; (b) implicit velocity stabilization. Dotted (resp., dashed) lines correspond to the fractional-step method using BDF2 (resp., BDF1). Solid lines mark first- and second-order slopes. Circle markers indicate $L^{2}$-norm errors on the velocity and square markers $L^{2}$-norm errors on the pressure. In (c) all the curves are collected in one graph for comparison. Here, the dash-dot curve indicates the BDF1 scheme, and dash-dot-dot curve indicates the BDF2 scheme, both with explicit treatment of the velocity stabilization.

driven by the body forces $f=u(x, y)\left(g^{\prime}(t)+2 g(t) / \mathrm{Re}\right)$. Dirichlet boundary conditions are imposed on the velocities on $\partial \Omega$ according to $(57)$. We have performed computations using the fractional-step method and BDF1 time stepping or BDF2 time stepping combined with either explicit or implicit treatment of the velocity stabilization in each case. In the implicit case we have set $\tau=h \in\left\{0.1 / 2^{i}\right\}_{i=0}^{3}$, whereas in the explicit case we have set $\tau=\operatorname{Co} \beta_{\Omega}^{-1} h$ with Co fixed (approximately) to the largest time step for which the scheme was stable, namely, $(\tau, h) \in\left\{\left(2.5 \cdot 10^{-2} / 2^{i}, 0.2 / 2^{i}\right)\right\}_{i=0}^{3}$. The results are reported in Figure 1. Observe that the points on the graphs correspond to the same meshes in the two cases. The values on the $\mathrm{x}$-axis correspond to time step sizes and those on the y-axis to the $L^{2}$-errors on the velocity (circles) and the pressure (squares). The curves corresponding to BDF1 time discretization are dashed and the curves corresponding to BDF2 time discretization are dotted. It follows from the graphics that in the case of BDF1, the reduced time step imposed by the explicit treatment of the stabilization also improves accuracy. The curves have the same slope in the explicit and implicit case, but the errors are approximately a factor three smaller and the time step differs by a factor of four. This shows that for the lowest-order time discretization, the explicit method is expected to be substantially cheaper than the fully implicit one if a specific accuracy has to be obtained. The situation is less clear for the second-order scheme where also the time step is taken four times smaller in the explicit case, but the resulting reduction in error is only a factor of two. 


\subsection{Flow around a cylinder at $\mathrm{Re}=10^{2}$}

Here we consider the classical benchmark proposed in [22] and we refer to that work for details on the configuration. The problem consists in the computation of the flow around a cylinder at Reynolds $10^{2}$, and the benchmark quantities that we consider are the $\operatorname{drag} C_{\mathrm{D}}$ and the lift $C_{\mathrm{L}}$ on the cylinder. We compare the fractional-step projection scheme using implicit treatment of the

\begin{tabular}{|cc|cc|}
\cline { 3 - 4 } \multicolumn{1}{c|}{} & \multicolumn{2}{c|}{ BDF2 monolithic } \\
\hline NDOF & $\tau$ & $C_{\mathrm{D}}$ & $C_{\mathrm{L}}$ \\
\hline $3 \cdot 2886$ & $10^{-2}$ & 3.34 & 1.12 \\
$3 \cdot 11055$ & $5 \cdot 10^{-3}$ & 3.27 & 1.05 \\
$3 \cdot 44540$ & $2.5 \cdot 10^{-3}$ & 3.24 & 1.03 \\
\hline & lower ref. & 3.22 & 0.99 \\
& upper ref. & 3.24 & 1.01 \\
\hline
\end{tabular}

Table 1: Computed drag and lift using the BDF2 monolithic solver.

\begin{tabular}{|cc|cc|cc|}
\cline { 3 - 6 } \multicolumn{1}{l|}{} & \multicolumn{2}{c|}{ BDF1 monolithic } & \multicolumn{2}{c|}{ BDF1 fractional-step } \\
\hline NDOF & $\tau$ & $C_{D_{\max }}$ & $C_{L_{\max }}$ & $C_{D_{\max }}$ & $C_{L_{\max }}$ \\
\hline $3 \cdot 2886$ & $5 \cdot 10^{-3}$ & 3.27 & 1.04 & 3.28 & 1.13 \\
$3 \cdot 11055$ & $2.5 \cdot 10^{-3}$ & 3.25 & 1.07 & 3.26 & 1.09 \\
$3 \cdot 44540$ & $1.25 \cdot 10^{-3}$ & 3.24 & 1.05 & 3.24 & 1.06 \\
\hline & lower ref. & 3.22 & 0.99 & 3.22 & 0.99 \\
& upper ref. & 3.24 & 1.01 & 3.24 & 1.01 \\
\hline
\end{tabular}

Table 2: Comparison of the computed drag and lift using the BDF1 monolithic and fractionalstep solvers.

velocity stabilization with the fully implicit monolithic scheme using either BDF1 or BDF2 for the time discretization. We have used three unstructured computational meshes with 2886, 11055 and 44540 vertices. Note that smaller time steps have been considered for the BDF1 schemes. The results are reported in Tables 1 and 2, As expected, the BDF2 scheme delivers comparable accuracy with larger time steps. We can also observe that the BDF1 projection scheme provides practically the same results as the BDF1 monolithic scheme for sufficiently small time steps.

\subsection{Navier-Stokes mixing layer at $\mathrm{Re}=10^{4}$}

Finally we propose a qualitative study of a Kelvin-Helmholtz shear layer. This test was proposed in [19] as a model problem for 2D turbulence and was shown to have the characteristic $k^{-3}$ decay of the power spectrum. The problem has a smooth solution, but the Sobolev norm of the exact solution is large. In particular, the velocity gradient is large in the shear layer and the problem is known to be very sensitive to perturbations of initial data and numerical viscosity. For other numerical experiments on this test case see [11, 16, 3]. A schematic illustration of the problem setup is presented in Figure 2. The computational domain $\Omega$ is the unit square, $\boldsymbol{u}_{\infty}=1, \beta_{\Omega}=\frac{1}{28}$, and the viscosity is set to $\nu=3.571 \cdot 10^{-6}$. The objective is to explore whether the use of the second-order time discretization remains stable while delivering the expected improved accuracy and to study how the splitting affects the approximation accuracy. As reference solutions we use computations from a monolithic solver with continuous interior penalty stabilization taken from [3]. The time step is $\tau=1.5625 \cdot 10^{-3}$ (as in [3]). 


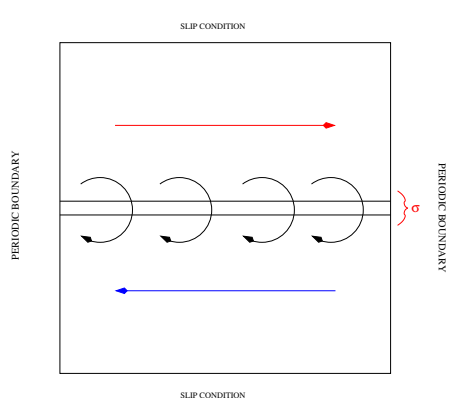

Figure 2: Computational configuration for the Kelvin-Helmholtz shear layer instability
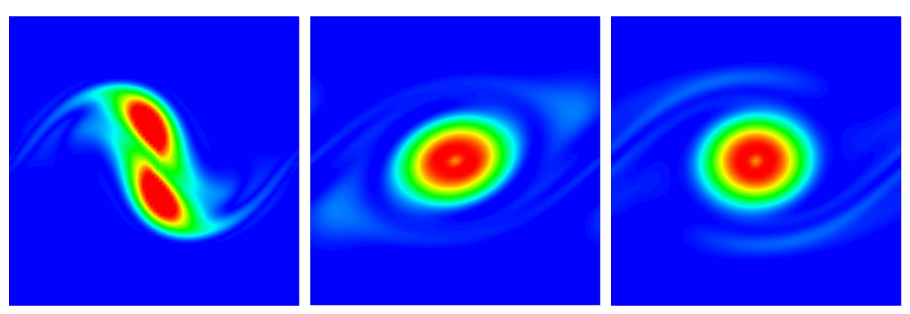

(a) Fractional-step BDF1, $k=1,80 \times 80$ mesh
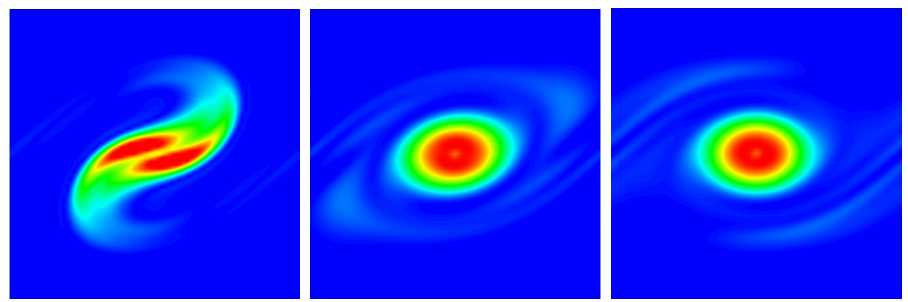

(b) Fractional-step BDF2, $k=1,80 \times 80$ mesh
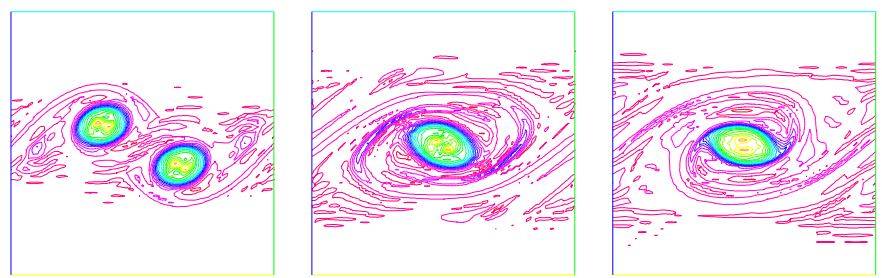

(c) Monolithic BDF2, , $k=1,80 \times 80$ mesh

Figure 3: Comparison of fractional-step BDF1 (a), BDF2 (b), and monolithic BDF2 time scheme (c); time levels, from left to right, $t=80,120,140$; computational mesh: $80 \times 80$; piecewise affine approximation.

Figure 3 shows a comparison between the solutions obtained using the fractional-step method combined with either BDF1 (a) or BDF2 (b) on a mesh with $80 \times 80$ elements using piecewise affine approximation. We present a series of snapshots at the non-dimensional times $t=80,120,140$. In Figure 3p (c) we report the reference solution obtained using BDF2 time discretization and a monolithic solver using the same space discretization and at the same time levels. On this 


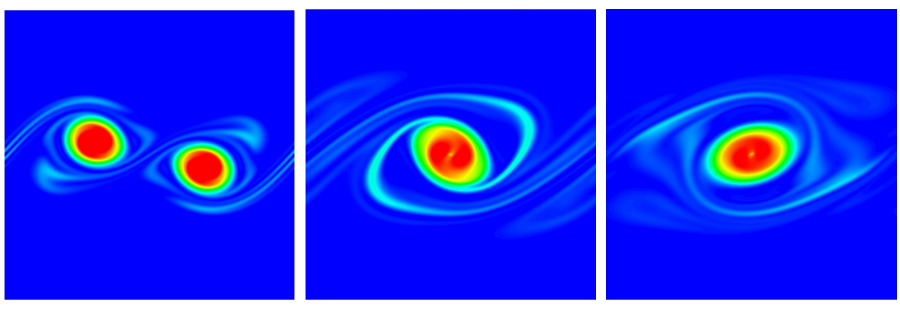

(a) Fractional-step BDF1, $k=1,320 \times 320$ mesh
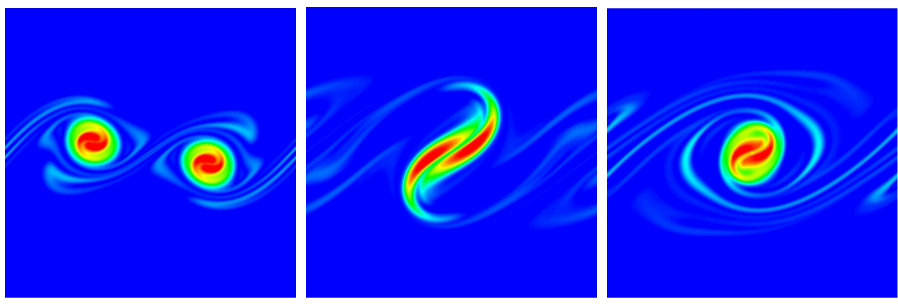

(b) Fractional-step BDF2, $k=1,320 \times 320$ mesh
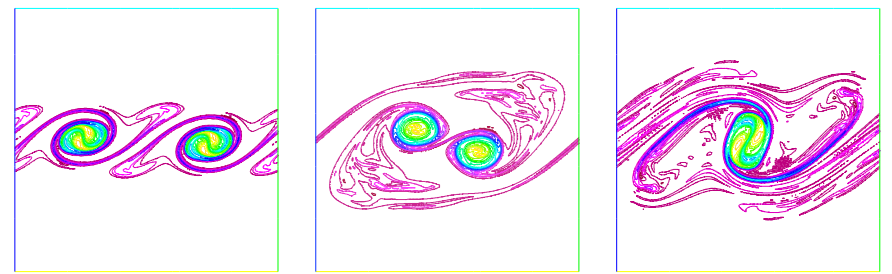

(c) Monolithic BDF2, $k=1,320 \times 320$ mesh
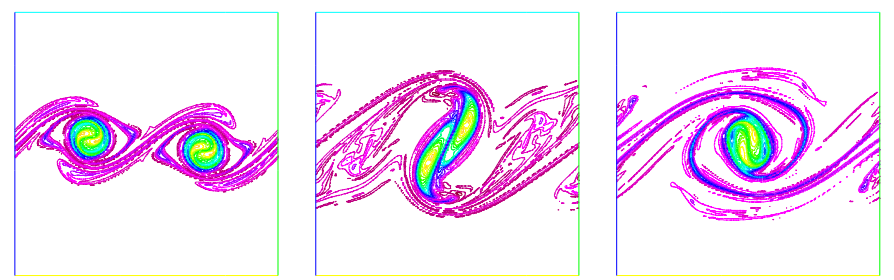

(d) Monolithic BDF2, $k=2,160 \times 160$ mesh

Figure 4: Comparison of fractional-step BDF1 (a), BDF2 (b) ; time levels, from left to right, $t=80,120,140$; computational mesh: $320 \times 320$; piecewise affine approximation. Monolithic computations: $\mathrm{BDF} 2 ; 320 \times 320$; piecewise affine approximation (c); $\mathrm{BDF} 2 ; 160 \times 160$; piecewise quadratic approximation (d).

coarse scale, the two fractional-step solutions appear to be of similar quality. The two vortices are merging in the first snapshot at $t=80$. The solution obtained using the monolithic solver on the other hand has not yet entered the transition phase in the first snapshot. A possible explanation of this is that the fractional-step method is more dissipative on coarse meshes, since it is known that excessive dissipation tends to speed up the transition sequence.

In Figure 4 we then consider the same sequence of snapshots on the finest mesh with $320 \times 320$ elements. In Figures $4(\mathrm{a})-(\mathrm{b})$ we present the snapshots of the fractional-step method using 
BDF1 and BDF2 respectively. Figures 4(c)-(d) report snapshots of solutions obtained using the monolithic solver on a $320 \times 320$ mesh and piecewise affine approximation (c) and on a $160 \times 160$ mesh and piecewise quadratic approximation $(d)$. In this case, the improved detail of the fractional-step method using BDF2 compared to the one using BDF1 can be clearly seen. On this resolution the fractional-step solutions using BDF2 has similar qualitative behaviour as those obtained using the monolithic scheme with BDF2.

\section{Acknowledgment}

This work was initiated during the Banff International Research Station workshop "Nonstandard Discretizations for Fluid Flows". The support from BIRS is gratefully acknowledged.

\section{References}

[1] R. Becker and M. Braack. A two-level stabilization scheme for the Navier-Stokes equations. In Numerical mathematics and advanced applications, pages 123-130. Springer, Berlin, 2004.

[2] M. Braack, E. Burman, V. John, and G. Lube. Stabilized finite element methods for the generalized Oseen problem. Comput. Methods Appl. Mech. Engrg., 196(4-6):853-866, 2007.

[3] E. Burman. Interior penalty variational multiscale method for the incompressible NavierStokes equation: monitoring artificial dissipation. Comput. Methods Appl. Mech. Engrg., 196(41-44):4045-4058, 2007.

[4] E. Burman, M. A. Fernández, and P. Hansbo. Continuous interior penalty finite element method for Oseen's equations. SIAM J. Numer. Anal., 44(3):1248-1274 (electronic), 2006.

[5] E. Burman and M.A. Fernández. Continuous interior penalty finite element method for the time-dependent Navier-Stokes equations: space discretization and convergence. Numer. Math., 107(1):39-77, 2007.

[6] E. Burman and P. Hansbo. Edge stabilization for Galerkin approximations of convectiondiffusion-reaction problems. Comput. Methods Appl. Mech. Engrg., 193:1437-1453, 2004.

[7] T. Chacón Rebollo, M. Gómez Mármol, and M. Restelli. Numerical analysis of penalty stabilized finite element discretizations of evolution Navier-Stokes equations. J. Sci. Comput., 63(3):885-912, 2015.

[8] R. Codina. Stabilization of incompressibility and convection through orthogonal sub-scales in finite element methods. Comput. Methods Appl. Mech. Engrg., 190(13-14):1579-1599, 2000 .

[9] R. Codina and S. Badia. On some pressure segregation methods of fractional-step type for the finite element approximation of incompressible flow problems. Comput. Methods Appl. Mech. Engrg., 195(23-24):2900-2918, 2006.

[10] A. Ern and J.-L. Guermond. Weighting the edge stabilization. SIAM J. Numer. Anal., 51(3):1655-1677, 2013.

[11] V. Gravemeier, W.A. Wall, and E. Ramm. Large eddy simulation of turbulent incompressible flows by a three-level finite element method. Internat. J. Numer. Methods Fluids, 48(10):1067-1099, 2005. 
[12] J.-L. Guermond. Stabilization of Galerkin approximations of transport equations by subgrid modeling. Math. Model. Numer. Anal. (M2AN), 33(6):1293-1316, 1999.

[13] J.-L. Guermond, A. Marra, and L. Quartapelle. Subgrid stabilized projection method for 2D unsteady flows at high Reynolds numbers. Comput. Methods Appl. Mech. Engrg., 195(4447):5857-5876, 2006.

[14] J. L. Guermond, P. Minev, and Jie Shen. An overview of projection methods for incompressible flows. Comput. Methods Appl. Mech. Engrg., 195(44-47):6011-6045, 2006.

[15] J.-L. Guermond and L. Quartapelle. On the approximation of the unsteady Navier-Stokes equations by finite element projection methods. Numer. Math., 80:207-238, 1998.

[16] V. John. An assessment of two models for the subgrid scale tensor in the rational LES model. J. Comput. Appl. Math., 173(1):57-80, 2005.

[17] C. Johnson, U. Nävert, and J. Pitkäranta. Finite element methods for linear hyperbolic problems. Comput. Methods Appl. Mech. Engrg., 45(1-3):285-312, 1984.

[18] C. Johnson and J. Pitkäranta. An analysis of the discontinuous Galerkin method for a scalar hyperbolic equation. Math. Comp., 46(173):1-26, 1986.

[19] M. Lesieur, C. Staquet, P. Le Roy, and P. Comte. The mixing layer and its coherence examined from the point of view of two-dimensional turbulence. J. Fluid Mech., 192:511$534,1988$.

[20] J. Nitsche. Über ein Variationsprinzip zur Lösung von Dirichlet-Problemen bei Verwendung von Teilräumen, die keinen Randbedingungen unterworfen sind. Abh. Math. Sem. Univ. Hamburg, 36:9-15, 1971. Collection of articles dedicated to Lothar Collatz on his sixtieth birthday.

[21] A. Prohl. Projection and quasi-compressibility methods for solving the incompressible NavierStokes equations. Advances in Numerical Mathematics. B. G. Teubner, Stuttgart, 1997.

[22] S. Turek and M. Schäfer. Benchmark computations of laminar flow around cylinder, chapter Flow Simulation with High-Performance Computers II. Vieweg, 1996. 


\section{Contents}

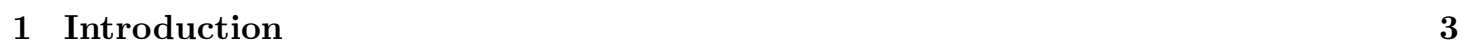

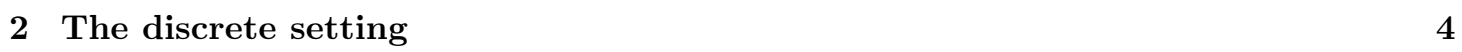

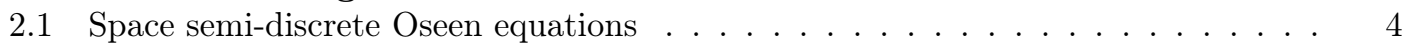

2.2 Analysis tools $\ldots \ldots \ldots \ldots \ldots \ldots \ldots \ldots$

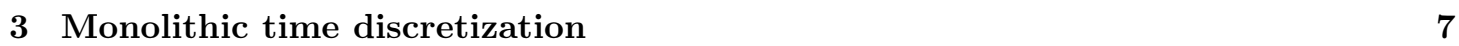

4 Fractional-step time discretization using pressure projection 9

4.1 Stability ............................ 10

4.2 Implicit or explicit of stabilization . . . . . . . . . . . . . . . . . . . . . . . 11

4.3 Error analysis $\ldots \ldots \ldots \ldots \ldots \ldots \ldots \ldots$

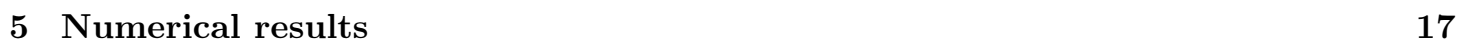

$5.1 \quad$ Academic $2 \mathrm{D}$ solution at $\mathrm{Re}=10^{5} \ldots \ldots \ldots \ldots$. . . . . . . . . . 18

$5.2 \quad$ Flow around a cylinder at $\mathrm{Re}=10^{2} \quad \ldots \ldots \ldots \ldots$. . . . . . . . . . . . . 19

$5.3 \quad$ Navier-Stokes mixing layer at $\mathrm{Re}=10^{4} \ldots \ldots \ldots \ldots$ 


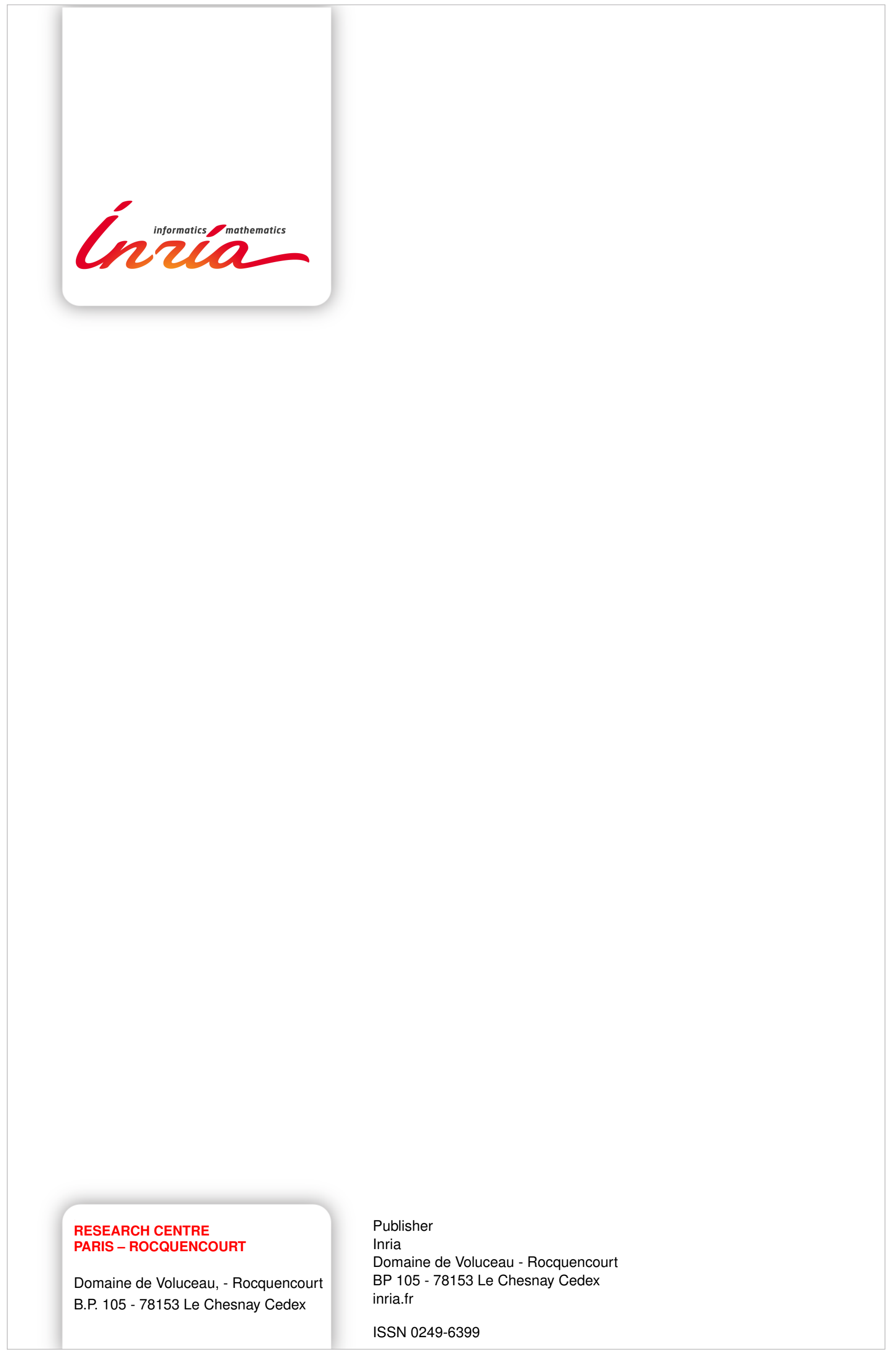

